\title{
VARDA (VARved sediments DAtabase) - providing and connecting proxy data from annually laminated lake sediments
}

\author{
Arne Ramisch, Alexander Brauser, Mario Dorn, Cecile Blanchet, Brian Brademann, Matthias Köppl, \\ Jens Mingram, Ina Neugebauer, Norbert Nowaczyk, Florian Ott, Sylvia Pinkerneil, Birgit Plessen, \\ Markus J. Schwab, Rik Tjallingii, and Achim Brauer
}

GFZ German Research Center for Geoscience, Section Climate Dynamics and Landscape Evolution, Telegrafenberg, 14473 Potsdam, Germany

Correspondence: Arne Ramisch (arne.ramisch@gfz-potsdam.de)

Received: 5 March 2020 - Discussion started: 30 March 2020

Revised: 27 July 2020 - Accepted: 3 August 2020 - Published: 25 September 2020

\begin{abstract}
Varved lake sediments provide climatic records with seasonal to annual resolution and low associated age uncertainty. Robust and detailed comparison of well-dated and annually laminated sediment records is crucial for reconstructing abrupt and regionally time-transgressive changes as well as validation of spatial and temporal trajectories of past climatic changes. The VARved sediments DAtabase (VARDA) presented here is the first data compilation for varve chronologies and associated palaeoclimatic proxy records. The current version 1.0 allows detailed comparison of published varve records from 95 lakes. VARDA is freely accessible and was created to assess outputs from climate models with high-resolution terrestrial palaeoclimatic proxies. VARDA additionally provides a technical environment that enables us to explore the database of varved lake sediments using a connected data model and can generate a state-of-the-art graphic representation of a multisite comparison. This allows the reassessment of existing chronologies and tephra events to synchronize and compare even distant varved lake records. Furthermore, the present version of VARDA permits the exploration of varve thickness data. In this paper, we report in detail on the data-mining and compilation strategies for the identification of varved lakes and assimilation of high-resolution chronologies, as well as the technical infrastructure of the database. Additional palaeoclimatic proxy data will be provided in forthcoming updates. The VARDA graph database and user interface can be accessed online at https://varve.gfz-potsdam.de (last access: 15 September 2020), all datasets of version 1.0 are available at https://doi.org/10.5880/GFZ.4.3.2019.003 (Ramisch et al., 2019).
\end{abstract}

1
A major challenge in simulating climate change is validating model outputs with palaeoclimatic data. Model-data com- parisons on regional to global scales require the integration of palaeoclimatic data from single sites into multisite networks (e.g. Franke et al., 2017). Annually laminated lake sediments provide reliable data for such networks because they of- fer palaeoclimatic information in high temporal resolution with low associated age uncertainty. Due to their annual to seasonal resolution, multisite networks of varved lake sed- iments enable investigations of abrupt and regionally time-

transgressive climate change on the continents (e.g. Lane et al., 2013; Rach et al., 2014), which are fundamental to understanding past climates, especially that of the last glacial cycle (Clement and Peterson, 2008), and to better assess spatial and temporal trajectories of future climate changes. Networks of varved lake sediments also provide means to test contrasted proxy responses to climate change (e.g. Ott et al., 2017; Ramisch et al., 2018; Roberts et al., 2016), further enhancing the robustness of palaeoclimatic reconstructions. However, despite their usefulness for the generation of highly resolved multisite networks, a global synthesis of varve-related palaeoclimatic data is still not available. 
Various data providers have been developed which offer free access to palaeoclimatic and palaeoenvironmental information including high-resolution terrestrial archives. These include (1) large-scale data repositories, such as PANGAEA (http://www.pangaea.de, last access: 15 September 2020), the National Oceanic and Atmospheric Administration's (NOAA) World data service for palaeoclimatology archives (http://www.ncdc.noaa.gov, last access: 15 September 2020) and Neotoma (http://www.neotomadb.org, last access: 15 September 2020, Williams et al., 2018), and (2) proxy- or time-slice-specific databases like the ACER (Sánchez Goñi et al., 2017), the European Pollen database (Fyfe et al., 2009), the SISAL database (Atsawawaranunt et al., 2018), or the PAGES2k Global 2000 Year Multiproxy Database (Pages 2k consortium, 2017). However, the distribution of information between data providers makes a custom generation of multisite networks from varved sediments inefficient and time-consuming. Moreover, continuous geochronological development results in frequent updates of fundamental methods such as calibration curves (e.g. Reimer et al., 2004, 2009, 2013) and age-depth modelling algorithms (e.g. Bronk Ramsey, 2008; Blaauw and Christen, 2011). Incorporating such changes into existing varve-related datasets requires an interactive approach that is not offered by fixed data structures of standard relational database management systems. To overcome these limitations, we developed a new and state-of-the-art graph database especially (but not exclusively) for varved sediment records. The database was developed within the German climate modelling initiative PalMod (Latif et al., 2016), to validate the output of comprehensive Earth system models with reliable proxy data from terrestrial and marine (Jonkers et al., 2020) archives. We compiled all available and published varved sediment records and developed criteria for how these data are integrated in this database.

\section{Data and methods}

\subsection{Data mining}

We assessed varve-related publications aided by the literature database of the PAGES varve working group (http://www.pastglobalchanges.org/download/docs/

working_groups/vwg/Varvepublications.pdf, last access: 15 September 2020) to identify lake archives exhibiting varved sediments and to compile suitable core-related palaeoclimatic proxy time series. A comprehensive set of lake sediment records was identified, for which proxy data from continuous or floating varve sequences were previously published. All data were collected as raw data from freely available online sources, either from online data repositories (PANGAEA, NOAA, and Neotoma) or data archives within the supplementary materials section of online publications. For a permanent and definite assignment of the compiled datasets within the database to their respective original pub- lication, the digital object identifier (DOI) of the publication or the data provider (if available) was additionally collected and stored.

\subsection{Data compilation}

To ensure an unambiguous identification of a lake record corresponding to a given dataset, we collected and reviewed the required information of lake names and geographic coordinates from the published literature. Table 1 lists required and additional information for lake records included in VARved sediments DAtabase (VARDA). To facilitate searches for lakes in an alphabetically ordered list, the string "Lake" was removed from the name if the string appeared in the beginning of the lake name (e.g. "Lake Ammersee" was changed to "Ammersee"). However, exceptions were made if the string "Lake" is an essential feature of the lake name (e.g. "Lake of the Clouds") or if the reference is in non-English language (e.g. "Lac d'Annecy"). Lake locations were stored as WGS84-referenced geographical coordinates in decimal degrees with four decimal places, which corresponds to a precision of $\sim 10 \mathrm{~m}$. This even allows a reliable location of small lakes with a surface area $<1$ ha and is especially useful for dense lake distributions common in large lake districts such as those in Canada or Scandinavia. Since the required precision was not available in most publications, we reassessed the published geographical location using ArcGIS and Google Earth. All lake locations refer to the approximate lake centre and are independent from coring locations.

Sediment composite profiles that were collected from primary literature sources (see Table 2) only require a unique identifier (e.g. MON for Lago Grande di Monticchio) within the VARDA database that links a profile to a corresponding lake (Table 2). Additional information encompasses the geographical coordinates of coring location (fields: latitude, longitude), coring methods (e.g. piston corer), a coring date, water depths at the core location, and the total length of the sediment composite profile with an upper (field: depth start) and lower (field: depth end) depth.

\subsubsection{Lake and sediment composite profile meta-information}

The data compilation followed the basic strategy of collecting proxy data associated with a published sediment composite profile and information about age-depth models and event layers. A sediment composite profile may either consist of a single core section or several overlapping core sections combined to a composite profile. The depth scale within a sediment composite profile is referred to as composite depth. Since data and meta-information availability greatly varied in between different publications, we classified the available information into required and additional information. The category required encompasses all information that is necessary to (a) associate a proxy value at a given depth in a 
Table 1. VARDA v01 data sheet for lake information (green field: required information; yellow field: additional information).

\begin{tabular}{lllllll}
\hline & & & & & & \\
\hline Attribute: & Name & Latitude Longitude & Elevation & Max depth & Surface area & Catchment area \\
Default units: & String & Decimal degrees (four-digit scale) & ma.s.l. & $\mathrm{m}$ & $\mathrm{m}^{2}$ & $\mathrm{~m}^{2}$ \\
\hline
\end{tabular}

Table 2. VARDA v01 data sheet for sediment composite profile information (green field: required information; yellow field: additional information).

\begin{tabular}{llllllll}
\hline & & & & & \\
\hline Attribute: & Label & Latitude Longitude & Coring method & Drill date \\
Default units: & String & $\begin{array}{l}\text { Decimal degrees } \\
\text { (four-digit scale) }\end{array}$ & String & dd/mm/year & $\mathrm{m}$ & $\begin{array}{l}\text { Depth start } \\
\mathrm{mm}\end{array}$ & $\begin{array}{l}\text { Depth end } \\
\mathrm{mm}\end{array}$ \\
\hline
\end{tabular}

sediment composite profile with a corresponding age and to (b) uniquely identify a lake, sediment composite profile, and original publication for a given dataset. The category additional encompasses all information that extends the data pool for more comprehensive analyses and therefore improves reproducibility, the ability to filter data by specific properties and, in addition, the quantification of methodological uncertainties. We converted all datasets to default units to provide standardized and thus inter-comparable data formats. Tables 1-7 provide an overview of data categories and required and additional information properties including the default units.

\subsubsection{Radiocarbon dates}

Uncalibrated radiocarbon measurements were collected from the published literature and adapted to the ${ }^{14} \mathrm{C}$ data reporting standards of Millard (2014). This allows efficient reassessments of published chronologies by calibration, agedepth modelling, and age uncertainty estimation (see Table 3 ). However, reporting standards are not yet fully adapted in the palaeoclimatic community, leading to variations in reported information and data gaps. The required information encompasses from left to right (i) the sampling depth (field: composite depth); (ii) the uncalibrated age (field: age uncalibrated); (iii) the associated measurement error (field: error); (iv) the error type (e.g. 1 sigma); and (v) the dated material (e.g. wood remains). The required sampling position refers to the depth within the sediment composite profile, whereas the sampling position within the individual core sections can be attributed as additional information. If available, we collected additional information on (i) the corresponding core section label (field core section); (ii) section depth (field: section depth); (iii) the lab code; (iv) $\delta^{13} \mathrm{C}$ data; (v) the measurement method (field: method), e.g. AMS ${ }^{14} \mathrm{C}$; (vi) the organic carbon content of a sample (field: \% C); and (vii) $\mathrm{C} / \mathrm{N}$ ratios.

\subsubsection{Age-depth models and chronologies}

Chronologies for varved lake sediments are commonly based on a combination of different dating methods (Brauer et al., 2014), such as varve counting, radiometric dating (e.g. ${ }^{14} \mathrm{C}$, ${ }^{137} \mathrm{Cs}$, or ${ }^{210} \mathrm{~Pb}$ ) and event age-equivalent dating (e.g. correlation to dated volcanic eruptions). Age-depth models provide the time frame for down-core sequences of sediment composite profiles and allow transformations of sediment proxy records into time series. Initially, most researchers constructed age-depth models by simple linear interpolation between individual chronological points. However, agedepth modelling algorithms such as the OxCal P-Sequence (Bronk-Ramsey, 2007) or Bacon (Blaauw and Christen, 2011) have become more common and perform more complex statistical interpolations.

VARDA version 1.0 includes published chronologies that are available in public data repositories. Tables 4 and 5 provide an overview of the required and additional metainformation for storing chronologies in VARDA and the resulting chronological data sheet, respectively. The required information includes a label for the associated sediment composite profile and the corresponding data and publication DOI. Additional information will enable rapid reassessments of original chronologies.

Additional information reports (i) on age uncertainty; (ii) presence, type, and age of anchor points for floating chronologies (e.g. sediment surface for continuous varve chronologies, ${ }^{14} \mathrm{C}$ dates, or elsewhere-dated tephra layers for floating chronologies); (iii) the applied dating methods (e.g. varve counting, radiometric dating, or event layers); (iv) the interpolation method (e.g. linear interpolation or Bayesian age-depth modelling, such as $\mathrm{OxCal} \mathrm{P}$-sequence or Bacon); (v) the applied ${ }^{14} \mathrm{C}$ calibration curve (e.g. IntCal09); and (vi) the resulting median resolution of the chronology.

Ideally, the chronological data sheet associates a given depth of a sediment composite profile to an age estimate and, if available, an uncertainty range expressed as minimum and 
Table 3. VARDA v01 data sheet for ${ }^{14} \mathrm{C}$ information (green field: required information; yellow field: additional information).

\begin{tabular}{|c|c|c|c|c|c|c|}
\hline Attribute: & $\begin{array}{l}\text { Sediment com- } \\
\text { posite profile }\end{array}$ & Lab code & Section depth & Composite depth & Age uncalibrated & Error \\
\hline Default units: & String & String & $\mathrm{mm}$ & $\mathrm{mm}$ & a BP & $\pm \mathrm{a}$ \\
\hline Attribute: & Error type & Dated material & $\delta^{13} \mathrm{C}$ & Method & $\% \mathrm{C}$ & $\mathrm{C} / \mathrm{N}$ ratio \\
\hline Default units: & 1 sigma $[\%]$ & String & $\%$ & String & $\%$ & dimensionless \\
\hline
\end{tabular}

Table 4. VARDA v01 data sheet for chronological meta-information (green field: required information; yellow field: additional information).

\begin{tabular}{|c|c|c|c|c|c|c|}
\hline Attribute: & $\begin{array}{l}\text { Sediment com- } \\
\text { posite profile }\end{array}$ & Data DOI & $\begin{array}{l}\text { Publication } \\
\text { DOI }\end{array}$ & Has uncertainty? & Uncertainty type & Anchored? \\
\hline Default units: & String & String & String & Boolean & String & Boolean \\
\hline Attribute: & $\begin{array}{l}\text { Anchor point } \\
\text { type }\end{array}$ & $\begin{array}{l}\text { Anchor point } \\
\text { age }\end{array}$ & Dating method & $\begin{array}{l}\text { Interpolation } \\
\text { method }\end{array}$ & $\begin{array}{l}{ }^{14} \mathrm{C} \text { calibration } \\
\text { curve }\end{array}$ & $\begin{array}{l}\text { Median } \\
\text { resolution }\end{array}$ \\
\hline Default units: & String & $\mathrm{aBP}$ & String & String & String & $\mathrm{a}$ \\
\hline
\end{tabular}

maximum estimate as additional information (2 sigma as a default). If depth information for a sediment composite profile was not provided, we either reconstructed an auxiliary composite depth by cumulative sums of continuous varve thickness measurements (if available) or excluded the corresponding chronology from the present data compilation because such time series without corresponding core depth are not updatable. The default depth scale unit was set to millimetres to avoid excessive decimal places in depth reporting. The default age scale unit was set to a BP (year before present) with $1950 \mathrm{CE}$ as zero age. The default age unit was restricted to annual precision and ages are reported in integer numbers (without usage of decimal places).

\subsubsection{Isochronous event layers}

Isochronous event layers provide precise tie points for the synchronization of proxy time series from regionally different locations and facilitate the construction of multisite networks. Furthermore, the identification of layers corresponding to dated events such as volcanic eruptions or geomagnetic excursions provide additional information for the construction of robust chronologies. For the first version of VARDA, we collected information on reported tephra layers in the sediment composite profiles included in the database. Table 6 provides an overview of required and additional information of published tephra layers in VARDA. The required information (composite depth, age, age error, and dating method) is essential for assigning a tephra layer to a given depth in a sed- iment composite profile and storing information on the age of the layer as it has been reported. Since standards for age reporting of tephra layers greatly vary in between different studies (e.g. uncalibrated vs. calibrated), information on the dating method and calibration are required for the field "dating method or calibration". The required field "dated in profile?" provides information on whether the age of the tephra layer originates from the corresponding sediment composite profile itself (field $=$ true) or if the age was adapted from the literature (field $=$ false). If the age was adapted from the literature, a DOI from the original publication is required. Further event layers such as geomagnetic excursions will be included in forthcoming versions of VARDA.

\subsubsection{Proxy data}

The technical infrastructure of VARDA is intended to attribute a down-profile record of palaeoclimatic proxy data to the corresponding chronology of the sediment composite profile. Therefore, the required information for proxy data sequences is the composite depth and a corresponding proxy measurement, while additional information further describes proxy specific measurement standards. We adapted the variable controlled vocabulary of the PaST thesaurus for proxy data (World Data Service for Paleoclimatology, https://www.ncdc.noaa.gov/ data-access/paleoclimatology-data/past-thesaurus, last access: 15 September 2020). Therefore, all proxy records will be broadly categorized into biological, sedimentological, 
Table 5. VARDA v01 chronology data sheet (green field: required information; yellow field: additional information).

\begin{tabular}{|c|c|c|c|c|c|}
\hline Attribute: & $\begin{array}{l}\text { Sediment composite } \\
\text { profile }\end{array}$ & depth & Age & Age min & Age max \\
\hline Default units: & String & $\mathrm{mm}$ & a BP & a BP & a BP \\
\hline
\end{tabular}

Table 6. VARDA v01 data sheet for tephra layers (green field: required information; yellow field: additional information).

\begin{tabular}{|c|c|c|c|c|c|c|}
\hline $\begin{array}{l}\text { Attribute: } \\
\text { Default units: }\end{array}$ & $\begin{array}{l}\text { Lab code } \\
\text { String }\end{array}$ & $\begin{array}{l}\text { Composite depth } \\
\mathrm{mm}\end{array}$ & $\begin{array}{l}\text { Age } \\
\text { a BP }\end{array}$ & $\begin{array}{l}\text { Error } \\
\pm \mathrm{a}\end{array}$ & \multicolumn{2}{|c|}{$\begin{array}{l}\text { Dating method or calibration } \\
\text { String }\end{array}$} \\
\hline Attribute: & $\begin{array}{l}\text { Correlated to } \\
\text { event }\end{array}$ & Source locality & $\begin{array}{l}\text { Major element } \\
\text { data available }\end{array}$ & $\begin{array}{l}\text { Trace element } \\
\text { data available }\end{array}$ & $\begin{array}{l}\text { Dated in } \\
\text { profile? }\end{array}$ & $\begin{array}{l}\text { Age transfer } \\
\text { reference }\end{array}$ \\
\hline Default units: & String & String & Boolean & Boolean & Boolean & DOI \\
\hline
\end{tabular}

and geochemical proxy data. In the present version of the database, we included varve thickness data that were found in public data repositories. Table 7 lists the required and additional information concerning varve thickness records. Further proxy data such as stable-isotope, pollen, or X-ray fluorescence (XRF) core scanning records will be included in forthcoming versions of VARDA.

\section{Database}

\subsection{Database design}

VARDA is intended to offer a flexible generation of multisite networks with complex data relations for storing and organizing the collected information. To store and organize datasets from varved lake archives, we use a graph database. Graph technology in computer science has evolved as part of the NoSQL movement (meaning "not only SQL"; SQL: Structured Query Language) and is based on graph theory, a mathematical concept of expressing objects as interconnected entities, which dates back to the early works of Leonard Euler in the 18th century (Euler, 1741). In contrast to fixed data schemes required by relational database management systems (RDBMS), a graph explicitly models relations between data by representing entities as nodes (or vertices) described by properties and connected through edges as shown in Fig. 1 (see also the property graph model). To categorize the nature of a particular entity, one or more labels can be added to the node. Edges can be distinguished by their type and may have properties just like nodes. The ability to add new labels, edges, and properties to any entity at all times enables developers to quickly adapt the data model to changing scientific or technical requirements. Neo4j's native query language Cypher is used to read and update the contents in the graph. It allows for an intuitive and flexible generation of queries that are short and readable even for complex patterns (many relationships, circular structures, variable-length paths).

The integration of palaeoenvironmental datasets from varved lakes into a graph database resulted in a flexible data structure, which allows for connected palaeoenvironmental datasets within a single lake as well as in between different lakes. Figure 1 illustrates the VARDA property graph model schematically and visualizes connections between nodes. The VARDA data model associates each lake with one or more sediment composite profiles, which are connected to one or more datasets. Datasets, in turn, are connected to a publication, a category (chronology, tephra layer, radiocarbon date, or varve thickness record in version 1.0) and various category-specific attributes (as listed in Tables 1-7) that further describe a dataset. All these connections provide the necessary meta-information to the actual data points, which are included in a given data set. Data points from the category tephra layer can additionally connect to an event that is described in more than one lake, e.g. the Laacher See tephra. The event node offers the possibility to connect datasets between different lakes for, e.g. synchronization.

\subsection{Application design}

VARDA provides fast access to palaeoclimatic data from varved lakes, irrespective of a user's technical background or operating system. Therefore, the user interface (UI) was designed to be intuitive and reactive with self-explanatory forms and components that immediately respond to the user's actions. It is implemented as an online service, which can be accessed permanently using a web browser.

Overall, the application consists of the web client, a serverside Neo4j graph database and an Application Programming Interface (API) for communication of the client with 
Table 7. VARDA v01 data sheet for varve thickness (Green field: required information, yellow field: additional information).

\begin{tabular}{lllllll}
\hline & & & & & \\
\hline Attribute: & $\begin{array}{l}\text { Sediment } \\
\text { composite } \\
\text { profile }\end{array}$ & $\begin{array}{l}\text { Varve } \\
\text { number }\end{array}$ & $\begin{array}{l}\text { Composite } \\
\text { depth } \\
\text { (varve top) }\end{array}$ & $\begin{array}{l}\text { Composite } \\
\text { depth } \\
\text { (varve } \\
\text { bottom) } \\
\mathrm{mm}\end{array}$ & Age & Varve thickness \\
Default unit: & String & Integer & $\mathrm{mm}$ & a BP & $\mathrm{mm}$ \\
\hline
\end{tabular}

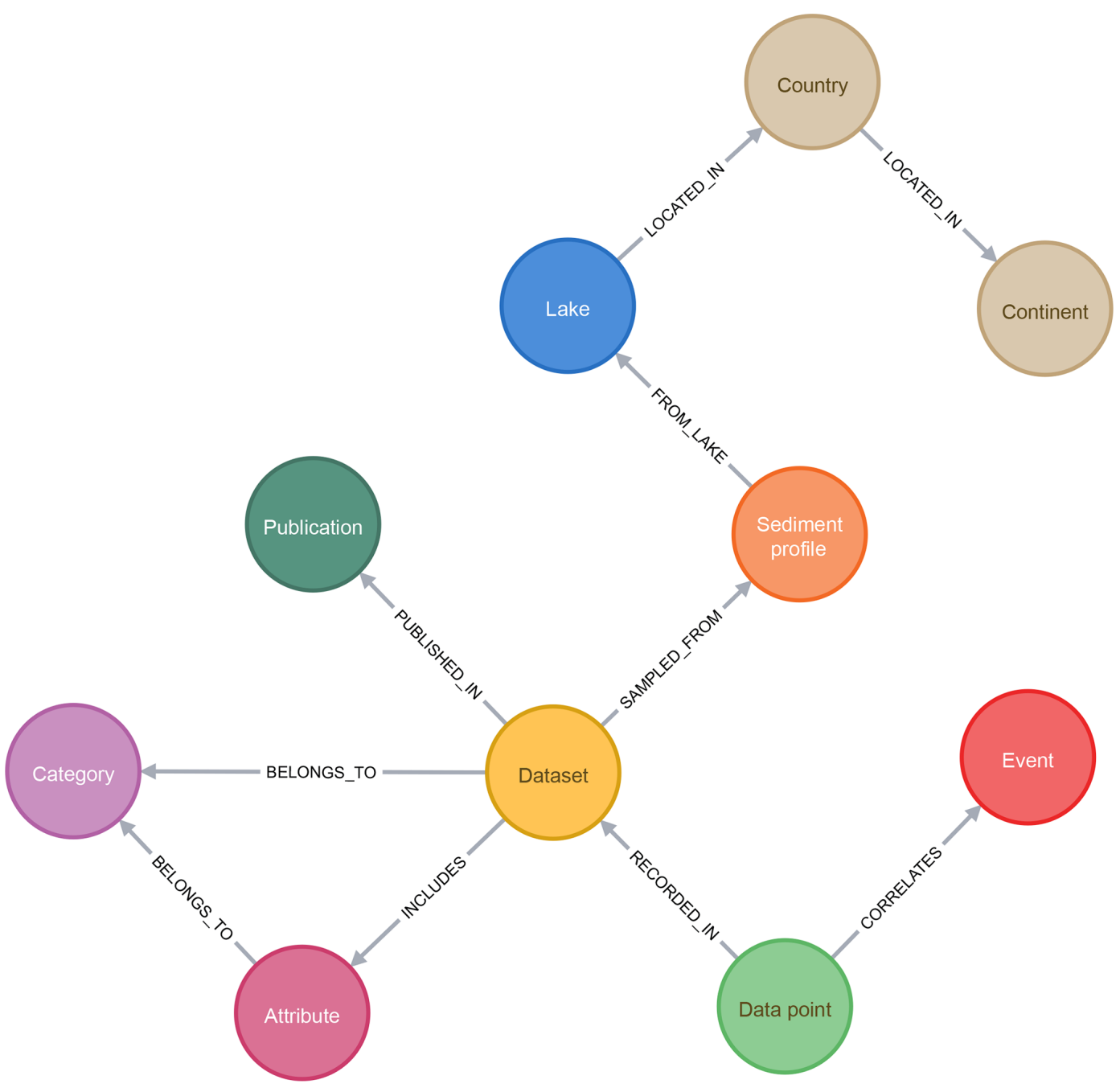

Figure 1. VARDA property graph model. Coloured circles represent nodes, and grey arrows represent edges between nodes. For an explanation, see the text.

the database. All software libraries that are integrated into VARDA have licenses that are free and permissive. The client is built with Vue.js, a JavaScript UI framework that has gained attention in the developer community since its launch in 2014 due to its versatility and runtime performance. Some features of VARDA integrate other well-documented third-party libraries, such as D3.js for data visualization and OpenLayers for rendering maps (e.g. from OSM) among vec- tor layers with spatial data. The client state (e.g. user data and entity cache) and any transactions with the database are handled with Apollo GraphQL, a framework for API communication and state management. The client's componentoriented architecture enables fast development of new features with little interference with existing modules. All lines of source code required by the client are checked, minified, and bundled using WebPack for use in the browser. 
Table 8. Identified lakes, updated geographic coordinates, and datasets included in VARDA 1.0. Letters indicate data availability in data repositories. Table also includes varved lake sites without publicly available data (without letters and references).

\begin{tabular}{|c|c|c|c|c|c|c|c|}
\hline Lake name & Latitude & Longitude & $\begin{array}{l}\text { Chrono- } \\
\text { logies }\end{array}$ & $\begin{array}{l}\text { Tephra } \\
\text { layers }\end{array}$ & ${ }^{14} \mathrm{C}$ & $\begin{array}{l}\text { Varve } \\
\text { thick. }\end{array}$ & References \\
\hline A & 83,0004 & $-75,4247$ & & & & & \\
\hline Ahvenainen & 60,8263 & 28,1254 & & & & & \\
\hline Albano & 41,7461 & 12,6695 & & & & & \\
\hline Alimmainen Savijärvi & 61,7442 & 24,4016 & & & & & \\
\hline Ammersee & 47,9983 & 11,1218 & A & & $\mathrm{B}$ & & $\begin{array}{l}\text { A: Grafenstein (1999); } \\
\text { B: Czymzik et al. (2013) }\end{array}$ \\
\hline Angulinao & 41,3500 & 114,3833 & & & & & \\
\hline Anterne & 45,9910 & 6,7983 & A & & & & A: Giguet-Covex et al. (2011) \\
\hline Arendsee & 52,8900 & 11,4759 & & & & & \\
\hline Arreo & 42,7784 & $-2,9911$ & & & & & \\
\hline Aspevatnet & 69,7503 & 19,9608 & & & A & & A: Bakke et al. (2005) \\
\hline Avigliana & 45,0654 & 7,3870 & & & & & \\
\hline Ayr Lake & 70,4590 & $-70,0860$ & A & & & A & A: Thomas et al. (2012) \\
\hline Baldeggersee & 47,1979 & 8,2614 & & & & & \\
\hline Barrine & $-17,2504$ & 145,6356 & & & A & & A: Head et al. (1994) \\
\hline Bear Lake (Canada) & 75,4838 & $-85,1900$ & & & & & \\
\hline Bear Lake (USA) & 41,9950 & $-111,3382$ & & A & & & A: Colman et al. (2009) \\
\hline Belau & 54,1006 & 10,2524 & A & $\mathrm{B}$ & $\mathrm{B}$ & & $\begin{array}{l}\text { A: Garbe-Schönberg et al. (1998); } \\
\text { B: Dörfler et al. (2012) }\end{array}$ \\
\hline Berrington Pool & 52,6605 & $-2,7042$ & & & & & \\
\hline Big Round Lake & 69,8648 & $-68,8548$ & A & & & A & A: Thomas and Briner (2008) \\
\hline Big Watab Lake & 45,5526 & $-94,4524$ & & & & & \\
\hline Bled & 46,3616 & 14,0953 & & A & & & A: Lane et al. (2011) \\
\hline Blue Lake & 68,0870 & $-150,4652$ & A & & A & A & A: Bird et al. (2008) \\
\hline Bosumtwi & 6,5014 & $-1,4113$ & & & & & \\
\hline Bourget & 45,7262 & 5,8673 & & & & & \\
\hline Bow Lake & 51,6644 & $-116,4486$ & & & A & & A: Leonard and Reasoner (1999) \\
\hline Bramant & 45,1999 & 6,1759 & & & A & & A: Guyard et al. (2007) \\
\hline Brownie Lake & 44,9676 & $-93,3243$ & & & & & \\
\hline Butrint & 39,7803 & 20,0313 & & & A & & A: Morellón et al. (2016) \\
\hline $\mathrm{C} 2$ & 82,8276 & $-77,9860$ & & & A & A & A: Lamoureux and Bradley (1996) \\
\hline Challa & $-3,3168$ & 37,7040 & A & & $\mathrm{B}$ & $\mathrm{C}$ & $\begin{array}{l}\text { A: Verschuren et al. (2009); } \\
\text { B: Blaauw et al. (2011); } \\
\text { C: Wolff et al. (2011) }\end{array}$ \\
\hline Cheakamus & 50,0080 & $-122,9179$ & & & & & \\
\hline Constance & 47,6017 & 9,4218 & & & & & \\
\hline Crawford Lake & 43,4684 & $-79,9488$ & A & & & & A: Yu and Eicher (1998) \\
\hline Crevice & 45,0006 & $-110,5784$ & & & A & & A: Whitlock et al. (2012) \\
\hline Czechowskie & 53,8740 & 18,2370 & A & $\mathrm{B} ; \mathrm{C}$ & & & $\begin{array}{l}\text { A: Dietze et al. (2019); } \\
\text { B: Wulf et al. (2016); } \\
\text { C: Wulf et al. (2013) }\end{array}$ \\
\hline Dead Sea & 31,5352 & 35,4909 & $\mathrm{~A} ; \mathrm{B}$ & & A & & $\begin{array}{l}\text { A: Migowski et al. (2004); } \\
\text { B: Neugebauer et al. (2015) }\end{array}$ \\
\hline Deep Lake & 47,6830 & $-95,3993$ & & & A & $\mathrm{B}$ & $\begin{array}{l}\text { A: Hu et al. (1997); } \\
\text { B: Hu et al. (1999) }\end{array}$ \\
\hline Diss Mere & 52,3754 & 1,1075 & & & & & \\
\hline Donard & 66,6625 & $-61,7875$ & A & & $\mathrm{B}$ & $\mathrm{B}$ & $\begin{array}{l}\text { A: Moore et al. (2001); } \\
\text { B: Moore et al. (2001) }\end{array}$ \\
\hline DV09 & 75,5744 & $-89,3094$ & A & & A & A & $\begin{array}{l}\text { A: Courtney Mustaphi and } \\
\text { Gajewski (2013) }\end{array}$ \\
\hline
\end{tabular}


Table 8. Continued.

\begin{tabular}{|c|c|c|c|c|c|c|c|}
\hline Lake name & Latitude & Longitude & $\begin{array}{l}\text { Chrono- } \\
\text { logies }\end{array}$ & $\begin{array}{l}\text { Tephra } \\
\text { layers }\end{array}$ & ${ }^{14} \mathrm{C}$ & $\begin{array}{l}\text { Varve } \\
\text { thick. }\end{array}$ & References \\
\hline East Lake & 74,8882 & $-109,5342$ & A & & & A & A: Cuven et al. (2011) \\
\hline Eklutna & 61,4053 & $-149,0259$ & A & A & A & A & A: Fortin et al. (2019) \\
\hline Elk Lake & 47,1891 & $-95,2179$ & & & A & $\mathrm{B}$ & $\begin{array}{l}\text { A: Smith et al. (1997); } \\
\text { B: Dean and Megard (1993) }\end{array}$ \\
\hline Ellesmere Mere & 52,9088 & $-2,8843$ & & & & & \\
\hline Erlongwan & 42,3026 & 126,3806 & & & & & \\
\hline Foy Lake & 48,1662 & $-114,3599$ & A & $\mathrm{B}$ & & & $\begin{array}{l}\text { A: Stone and Fritz (2006); } \\
\text { B: Shuman et al. (2009) }\end{array}$ \\
\hline Frängsjön & 64,0228 & 19,7376 & & & & & \\
\hline Frías & $-41,0617$ & $-71,7990$ & & & A & & A: Ariztegui et al. (2007) \\
\hline Frickenhäuser See & 50,4029 & 10,2373 & & & & & \\
\hline Fukami & 35,3256 & 137,8195 & & & & & \\
\hline Furskogstjärnet & 59,3802 & 12,0801 & & A & & & A: Zillén et al. (2002) \\
\hline Geneva & 46,4392 & 6,5164 & & & & & \\
\hline Glacier Lake & 40,0230 & $-105,5027$ & & & & & \\
\hline Gosciaz & 52,5829 & 19,3398 & & & & & \\
\hline Gölcük & 31,6270 & 40,6547 & & A & & & A: Sullivan (1988) \\
\hline Green Lake & 43,8110 & $-89,0002$ & & & & & \\
\hline Greifen & 47,3500 & 8,6794 & & & & & \\
\hline Grimselsee & 46,5680 & 8,3092 & & & & & \\
\hline Gropviken & 58,3376 & 16,6678 & & A & & & A: Macleod et al. (2014) \\
\hline Gyltigesjön & 56,7567 & 13,1754 & & & $\mathrm{~A} ; \mathrm{B}$ & & $\begin{array}{l}\text { A: Mellström et al. (2013); } \\
\text { B: Snowball et al. (2013) }\end{array}$ \\
\hline Hämelsee & 52,7596 & 9,3107 & & A & & & A: Jones et al. (2017) \\
\hline Hancza & 54,2647 & 22,8126 & A & & A & & A: Lauterbach et al. (2010) \\
\hline Hännisenlampi & 62,0750 & 30,2096 & & & & & \\
\hline Hector Lake & 51,5881 & $-116,3643$ & & A & A & & A: Leonard and Reasoner (1999) \\
\hline Hell's Kitchen Lake & 46,1868 & $-89,7025$ & & & & & \\
\hline Holzmaar & 50,1193 & 6,8787 & A & $\mathrm{B}$ & $\mathrm{B}$ & & $\begin{array}{l}\text { A: Zolitschka et al. (2000); } \\
\text { B: Prasad and Baier (2014) }\end{array}$ \\
\hline Hoya La Alberca & 20,3889 & $-101,2009$ & & & & & \\
\hline Hoya Rincón de Parangueo & 20,4311 & $-101,2495$ & & & A & & A: Park et al. (2010) \\
\hline Huron & 44,6418 & $-82,3580$ & & & & & \\
\hline Hvítárvatn & 64,6101 & $-19,8401$ & A & A & & $\mathrm{A} ; \mathrm{B}$ & $\begin{array}{l}\text { A: Larsen et al. (2011); } \\
\text { B: Larsen et al. (2013) }\end{array}$ \\
\hline Iceberg Lake & 60,7880 & $-142,9589$ & A & & $\mathrm{B}$ & $\mathrm{A} ; \mathrm{B}$ & $\begin{array}{l}\text { A: Loso (2008); } \\
\text { B: Diedrich and Loso (2012) }\end{array}$ \\
\hline Järlasjön & 59,3020 & 18,1515 & & & & & \\
\hline Judesjön & 62,8337 & 17,7728 & & & & & \\
\hline Jyväsjärvi & 62,2385 & 25,7771 & & & & & \\
\hline Kälksjön & 60,1531 & 13,0559 & & & & & \\
\hline Kallio Kourujärvi & 62,5600 & 27,0030 & A & $\mathrm{B}$ & & A & $\begin{array}{l}\text { A: Saarni et al. (2015a); } \\
\text { B: Kalliokoski et al. (2018) }\end{array}$ \\
\hline Kalliojärvi & 63,2261 & 25,3678 & A & & & A & A: Saarni et al. (2015b) \\
\hline Kassjön & 63,9254 & 20,0100 & & & & & \\
\hline Kissalammi & 61,2556 & 24,3549 & & & & & \\
\hline Koltjärnen & 62,9526 & 18,3043 & & & & & \\
\hline Kongressvatnet & 78,0212 & 13,9605 & & & & & \\
\hline Kortejärvi & 63,6236 & 28,9341 & & & & & \\
\hline Korttajärvi & 62,3373 & 25,6903 & & & & & \\
\hline Lac Brulé & 45,7192 & $-75,4422$ & A & & A & A & $\begin{array}{l}\text { A: Lafontaine-Boyer and } \\
\text { Gajewski (2014) }\end{array}$ \\
\hline
\end{tabular}


Table 8. Continued.

\begin{tabular}{|c|c|c|c|c|c|c|c|}
\hline Lake name & Latitude & Longitude & $\begin{array}{l}\text { Chrono- } \\
\text { logies }\end{array}$ & $\begin{array}{l}\text { Tephra } \\
\text { layers }\end{array}$ & ${ }^{14} \mathrm{C}$ & $\begin{array}{l}\text { Varve } \\
\text { thick. }\end{array}$ & References \\
\hline Lac d'Annecy & 45,8578 & 6,1717 & & & A & & A: Brauer and Casanova (2001) \\
\hline Lac Pavin & 45,4955 & 2,8877 & & & & & \\
\hline Etoliko & 38,4732 & 21,3248 & A & & $\mathrm{B}$ & A & $\begin{array}{l}\text { A: Koutsodendris et al. (2017); } \\
\text { B: Haenssler et al. (2013) }\end{array}$ \\
\hline Lago Buenos Aires & $-46,4900$ & $-72,0129$ & & A & & & A: Bendle et al. (2017) \\
\hline Laguna Potrok Aike & $-51,9608$ & $-70,3794$ & A & $\mathrm{B}$ & B & & $\begin{array}{l}\text { A: Kliem et al. (2013); } \\
\text { B: Haberzettl et al. (2007) }\end{array}$ \\
\hline Lake of the Clouds & 48,1426 & $-91,1122$ & & & & & \\
\hline Lampellonjärvi & 61,0737 & 25,0605 & & & & & \\
\hline Längsee & 46,7894 & 14,4242 & & A & & & A: Schmidt et al. (2002) \\
\hline Laukunlampi & 62,6682 & 29,1564 & & & & & \\
\hline Lavijärvi & 61,6333 & 30,5000 & & & & & \\
\hline Lehmilampi & 63,6283 & 29,1022 & A & & & A & A: Haltiahovi et al. (2007) \\
\hline Lillooet & 50,2425 & $-122,4973$ & & & & & \\
\hline Lind & 45,7504 & $-92,4354$ & & & & & \\
\hline Linné & 78,0463 & 13,8028 & & & & A & A: Werner et al. (2009) \\
\hline Loch Ness & 57,3000 & $-4,4500$ & & & & & \\
\hline Loe Pool & 50,0730 & $-5,2909$ & & & & & \\
\hline Lögurinn & 65,2507 & $-14,4649$ & & A & & & A: Striberger et al. (2010) \\
\hline Lower Murray Lake & 81,3328 & $-69,5510$ & A & & & A & A: Cook et al. (2008) \\
\hline Lower Mystic Lake & 42,4261 & $-71,1474$ & & & & & \\
\hline Lugano & 45,9203 & 8,9053 & & & & & \\
\hline Malawi & $-11,5486$ & 34,5376 & $\mathrm{~A} ; \mathrm{B}$ & & $\mathrm{C}$ & & $\begin{array}{l}\text { A: Sánchez Goñi et al. (2017); } \\
\text { B: Ivory et al. (2016); } \\
\text { C: Pilskaln and Johnson (1991) }\end{array}$ \\
\hline Mascardi & $-41,3157$ & $-71,5757$ & & & A & & A: Hajdas et al. (2003) \\
\hline McCarrons & 44,9981 & $-93,1131$ & & & & & \\
\hline Meerfelder Maar & 50,1010 & 6,7570 & A & $\mathrm{B} ; \mathrm{C}$ & $\mathrm{D}$ & $\mathrm{A} ; \mathrm{B} ; \mathrm{E} ; \mathrm{F}$ & $\begin{array}{l}\text { A: Martin-Puertas et al. (2012); } \\
\text { B: Engels et al. (2015); } \\
\text { C: Lane et al. (2015); } \\
\text { D: Brauer et al. (2000); } \\
\text { E: Brauer et al. (2008); } \\
\text { F: Litt et al. (2009) }\end{array}$ \\
\hline Mina & 45,8878 & $-95,4788$ & & & & & \\
\hline Mirror Lake & 62,0305 & $-128,2840$ & & & & & \\
\hline Mondsee & 47,8157 & 13,3819 & A & & B & & $\begin{array}{l}\text { A: Lauterbach et al. (2011); } \\
\text { B: Swierczynski et al. (2013) }\end{array}$ \\
\hline Montcortés & 42,3306 & 0,9951 & & & A & & A: Corella et al. (2010) \\
\hline Monticchio & 40,9313 & 15,6050 & $\mathrm{~A} ; \mathrm{B}$ & $\mathrm{C} ; \mathrm{D} ; \mathrm{E}$ & $\mathrm{F} ; \mathrm{G} ; \mathrm{H}$ & & $\begin{array}{l}\text { A: Martin-Puertas et al. (2014); } \\
\text { B: Allen et al. (1999); } \\
\text { C: Huntley et al. (1999); } \\
\text { D: Wulf et al. (2012); } \\
\text { E: Wulf et al. (2004); } \\
\text { F: Hajdas et al. (1997); } \\
\text { G: Watts (1996); } \\
\text { H: Zolitschka (1996) }\end{array}$ \\
\hline Mötterutstjärnet & 59,6394 & 12,6675 & & A & & & A: Zillén et al. (2002) \\
\hline Murray Lakes & 81,3555 & $-69,5436$ & & & & & \\
\hline Nar Gölü (Lake) & 38,3403 & 34,4560 & & & & & \\
\hline Nautajärvi & 61,8052 & 24,6782 & & & & & \\
\hline Nedre Heimredalsvatnet & 68,2990 & 13,6547 & & & A & & A: Balascio et al. (2011) \\
\hline Nedrefloen & 61,9306 & 6,8664 & & & A & & A: Vasskog et al. (2012) \\
\hline Nicolay Lake & 77,7670 & $-94,6529$ & & & & & \\
\hline
\end{tabular}


Table 8. Continued.

\begin{tabular}{|c|c|c|c|c|c|c|c|}
\hline Lake name & Latitude & Longitude & $\begin{array}{l}\text { Chrono- } \\
\text { logies }\end{array}$ & $\begin{array}{l}\text { Tephra } \\
\text { layers }\end{array}$ & ${ }^{14} \mathrm{C}$ & $\begin{array}{l}\text { Varve } \\
\text { thick. }\end{array}$ & References \\
\hline Nikkilänlampi & 63,1745 & 30,9479 & & & & & \\
\hline Ni no Megata & 39,9524 & 139,7284 & & A & & & A: Yamada et al. (2010) \\
\hline Nylandssjön & 62,9458 & 18,2826 & & & & & \\
\hline Oeschinen & 46,4984 & 7,7274 & A & & $\mathrm{A}$ & & A: Amann et al. (2015) \\
\hline Ogac & 62,8432 & $-67,3401$ & & & & & \\
\hline Ohrid & 41,0371 & 20,7181 & $\mathrm{~A} ; \mathrm{B} ; \mathrm{C} ; \mathrm{D}$ & $\mathrm{E} ; \mathrm{F}$ & $\mathrm{F}$ & & $\begin{array}{l}\text { A: Vogel et al. (2010a); } \\
\text { B: Wagner et al. (2008); } \\
\text { C: Francke et al. (2016); } \\
\text { D: Wagner et al. (2010); } \\
\text { E: Leicher et al. (2016); } \\
\text { F: Vogel et al. (2010b) }\end{array}$ \\
\hline Ojibway & 48,4739 & $-79,2801$ & & & & & \\
\hline Pääjärvi & 61,0625 & 25,1307 & & & & & \\
\hline Pavin & 45,4957 & 2,8879 & A & & $\mathrm{B}$ & & $\begin{array}{l}\text { A: Stebich et al. (2005); } \\
\text { B: Chassiot et al. (2016) }\end{array}$ \\
\hline Perespilno & 51,4269 & 23,5695 & & & & & \\
\hline Pettaquamscutt & 41,5030 & $-71,4506$ & & & A & & A: Hubeny et al. (2008) \\
\hline Pitkälampi & 62,2543 & 30,4679 & & & & & \\
\hline Plomo & $-47,0047$ & $-72,9122$ & A & & & & A: Elbert et al. (2015) \\
\hline Pohjajärvi & 62,8157 & 28,0332 & & & & & \\
\hline Polvijärvi & 63,1614 & 28,9700 & & & & & \\
\hline Prespa & 40,8967 & 21,0050 & & A; B & A & & $\begin{array}{l}\text { A: Wagner et al. (2012); } \\
\text { B: Wagner et al. (2010) }\end{array}$ \\
\hline Puyehue & $-40,6667$ & $-72,4667$ & & & $\mathrm{~A}$ & & A: Bertrand et al. (2008) \\
\hline Pyhäjärvi & 60,7167 & 26,0000 & & & & & \\
\hline Rehwiese & 52,4280 & 13,1996 & A & A & & A & A: Neugebauer et al. (2012) \\
\hline Rostherne Mere & 53,3543 & $-2,3862$ & & & & & \\
\hline Rõuge Suurjärv & 57,7282 & 26,9223 & & & & & \\
\hline RS29 & 73,1400 & $-95,2780$ & & & A & & A: Paull et al. (2017) \\
\hline Rudetjärn & 62,3662 & 16,9975 & & & & & \\
\hline Sacrower See & 52,4432 & 13,0991 & & $\mathrm{~A}$ & $\mathrm{~A}$ & & A: Enters et al. (2009) \\
\hline Saky & 45,1224 & 33,5612 & & & & & \\
\hline San Pueto & 41,2856 & 13,4080 & & & & & \\
\hline Sanagak Lake & 70,2095 & $-93,6355$ & & & & & \\
\hline Sarsjön & 64,0387 & 19,6008 & & & & & \\
\hline Sawtooth & 79,3494 & $-83,9235$ & & & & $\mathrm{~A}$ & A: Francus et al. (2002) \\
\hline Schleinsee & 47,6122 & 9,6348 & & A & & & A: Clark et al. (1989) \\
\hline Seebergsee & 46,5773 & 7,4433 & & & & & \\
\hline Sihailongwan & 42,2865 & 126,6019 & A & $\mathrm{A}$ & & & A: Mingram et al. (2018) \\
\hline Silvaplana & 46,4487 & 9,7923 & & & & & \\
\hline Skilak Lake & 60,4107 & $-150,3386$ & & & & & \\
\hline Soppensee & 47,0901 & 8,0803 & & $\mathrm{~A}$ & $\mathrm{~B}$ & & $\begin{array}{l}\text { A: Hajdas and Michczyński (2010); } \\
\text { B: Gierga et al. (2016) }\end{array}$ \\
\hline Sotkulampi & 61,4964 & 29,0894 & & & & & \\
\hline Starnberger See & 47,9000 & 11,3167 & & & & & \\
\hline Steel Lake & 46,9730 & $-94,6834$ & & & A & & A: Tlan et al. (2005) \\
\hline Storsjön & 63,2149 & 14,3146 & $\mathrm{~A}$ & & A & & A: Labuhn et al. (2018) \\
\hline Sugan Lake & 38,8667 & 93,9000 & A & & $\mathrm{B}$ & & $\begin{array}{l}\text { A: Zhang et al. (2009); } \\
\text { B: Zhou et al. (2009) }\end{array}$ \\
\hline Suigetsu & 35,5833 & 135,8833 & & $\mathrm{~A}$ & & & A: Smith et al. (2013) \\
\hline Suminko & 54,1841 & 17,7970 & & & & & \\
\hline Summit Lake & 59,6737 & $-135,0958$ & & & & & \\
\hline
\end{tabular}


Table 8. Continued.

\begin{tabular}{|c|c|c|c|c|c|c|c|}
\hline Lake name & Latitude & Longitude & $\begin{array}{c}\text { Chrono- } \\
\text { logies }\end{array}$ & $\begin{array}{l}\text { Tephra } \\
\text { layers }\end{array}$ & ${ }^{14} \mathrm{C}$ & $\begin{array}{l}\text { Varve } \\
\text { thick. }\end{array}$ & References \\
\hline Superior & 47,7508 & $-72,2719$ & $\mathrm{~A}$ & & & & A: O'Beirne et al. (2017) \\
\hline Szurpily & 54,2291 & 22,8978 & & & & & \\
\hline Taka-Killo & 61,0584 & 24,9477 & & & & & \\
\hline Tanganyika & $-5,8363$ & 29,5976 & $\mathrm{~A} ; \mathrm{B} ; \mathrm{C} ; \mathrm{D}$ & $\mathrm{E}$ & & & $\begin{array}{l}\text { A: Sánchez Goñi et al. (2017); } \\
\text { B: Tierney et al. (2010); } \\
\text { C: Tierney et al. (2008); } \\
\text { D: Tierney and Russell (2007); } \\
\text { E: Williamson et al. (1991) }\end{array}$ \\
\hline Tekapo & 35,0301 & $-108,9329$ & & & & & \\
\hline Teletskoye & 51,5914 & 87,6672 & & & A & & A: Rudaya et al. (2016) \\
\hline Tiefer See & 53,5946 & 12,5281 & A & B & & & $\begin{array}{l}\text { A: Dräger et al. (2016); } \\
\text { B: Wulf et al. (2016) }\end{array}$ \\
\hline Tõugjärv & 57,7386 & 26,9051 & & & & & \\
\hline Tougou-ike & 35,4775 & 133,8925 & & $\mathrm{~A}$ & & & A: Kato et al. (2003) \\
\hline Trübsee & 46,7942 & 8,3899 & & & & & \\
\hline Tuborg & 80,9500 & $-75,7667$ & & & & & \\
\hline Tutira & $-39,2238$ & 176,8923 & & $\mathrm{~A}$ & & & A: Eden and Page (1998) \\
\hline Upper Soper Lake & 62,9150 & $-69,8784$ & & & & & \\
\hline Valkiajärvi & 61,9048 & 23,8812 & & & & & \\
\hline Van & 38,6040 & 42,8763 & $\mathrm{~A}$ & & & & A: Pickarski et al. (2015) \\
\hline Vesijärvi & 61,1368 & 25,4732 & & & & & \\
\hline Victoria & 33,19833 & $-1,2317$ & $\mathrm{~A} ; \mathrm{B} ; \mathrm{C}$ & $\mathrm{D}$ & & & $\begin{array}{l}\text { A: Stager et al. (2005); } \\
\text { B: Stager et al. (2002); } \\
\text { C: Berke et al. (2012); } \\
\text { D: Lane et al. (2018) }\end{array}$ \\
\hline Vuolep Njakajaure & 68,3419 & 18,7808 & & & & & \\
\hline Waikopiro & $-39,2351$ & 176,8944 & & & & & \\
\hline Woserin & 53,6684 & 12,0263 & A & & & $\mathrm{A}$ & A: Czymzik et al. (2016) \\
\hline Xiaolongwan & 42,2999 & 126,3594 & & & & & \\
\hline Xinluhai & 31,8485 & 99,1129 & & & & & \\
\hline Yoa & 19,0576 & 20,5069 & & & & & \\
\hline Żabińskie & 54,1318 & 21,9836 & & A & & & A: Żarczyński et al. (2018) \\
\hline Zoñar & 37,4833 & $-4,6897$ & & & A & & A: Martín-Puertas et al. (2008) \\
\hline Zürichsee & 47,2513 & 8,6672 & & & & & \\
\hline
\end{tabular}

The web application offers a user interface with optional filters to explore and visualize multisite networks on demand (see Fig. 2). A universal search field (1 in Fig. 2) can be used to select filters either by region or proxy category. An interactive diagram ( 2 in Fig. 2) can be used to select a temporal filter by scrolling with the mouse or resizing the light-bluecoloured frame (3 in Fig. 2) underneath the main figure.

We add the iconic NGRIP oxygen-isotope $(\delta 180)$ record with the GICC05 chronology (Vinther et al., 2006; Rasmussen et al., 2006; Andersen et al., 2006; Svensson et al., 2005) as a temporal reference curve for the user. This curve is well-known in the palaeoclimate community and thus allows an easy recognition of the time interval covered by a lake record of interest. In the present version it does not allow precise correlations between lake records with the NGRIP curve because chronological uncertainties for the latter are not shown for visual clarity. Orange circles (4 in Fig. 2) cor- respond to tephra layers that have been identified in sediments of at least two archives. Clicking a circle enables (or disables) the respective filter. The results will be updated immediately on the map (5 in Fig. 2) and in the result list (6 in Fig. 2) below whenever any filters have been changed. Direct selection of a lake on the map or in the result list guides users to the lake detail view with a list of corresponding core datasets. In version 1.0 all datasets of interest can be downloaded in CSV format.

\section{Data inventory}

We identified 186 lakes from the published literature, which are described as exhibiting continuous or floating varve sequences in their sediments. We additionally included unvarved sediments from Lake Prespa (Europe), Lake Ohrid (Europe), Laguna Potrok Aike (South America), and Bear 


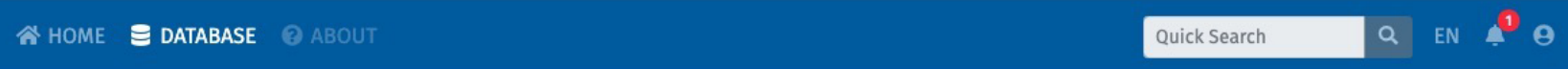

Q Filters to be used

1 Europe $\mathbf{x}$ Age within time span $\mathbf{x}$

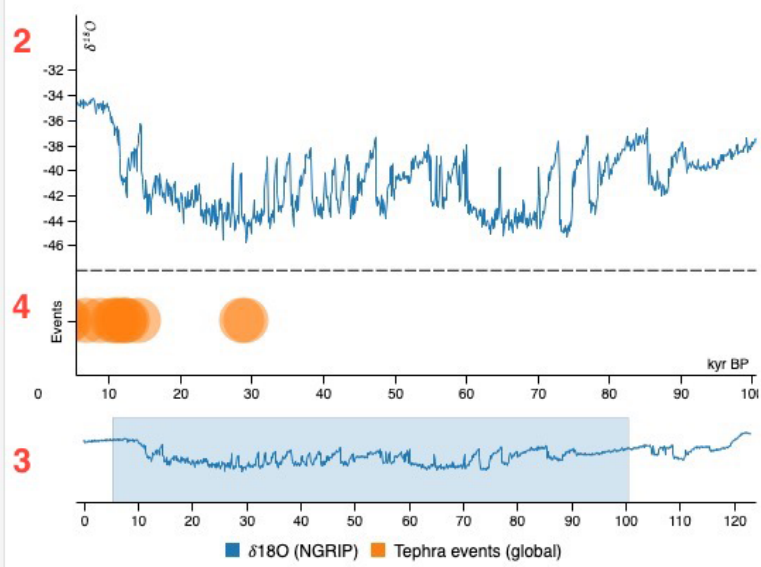

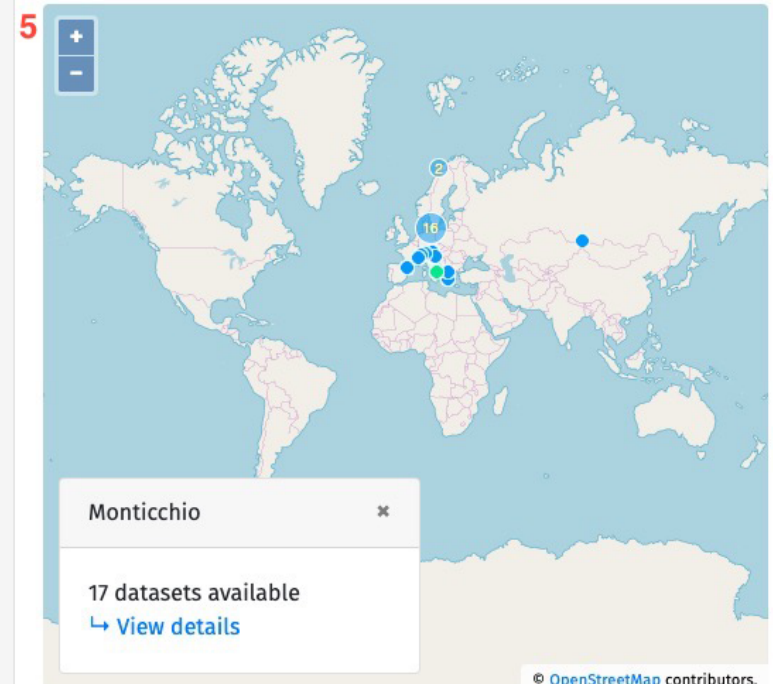

(i) OpenStreetMap contributors.

6

30 sites that match your criteria:

\begin{tabular}{|c|c|c|c|c|c|}
\hline Name & Country & Coordinates & & Datasets & \\
\hline Aspevatnet & NO & $69.75030^{\circ} \mathrm{N}$, & $19.96081^{\circ} \mathrm{E}$ & 1 & Details \\
\hline Belau & $\mathrm{DE}$ & $54.10056^{\circ} \mathrm{N}$, & $10.25236^{\circ} \mathrm{E}$ & 2 & Details \\
\hline Bled & SI & $46.36162^{\circ} \mathrm{N}$, & $14.09529^{\circ} \mathrm{E}$ & 1 & Details \\
\hline Czechowskie & PL & $53.87400^{\circ} \mathrm{N}$, & $18.23700^{\circ} \mathrm{E}$ & 2 & Details \\
\hline
\end{tabular}

Figure 2. Screenshot of the user interface in version 1.0, available online at https://varve.gfz-potsdam.de (last access: 15 September 2020). See text for explanation. (C) OpenStreetMap contributors 2019. Distributed under a Creative Commons BY-SA License.

Lake (North America) to the compilation due to their long continuous chronologies and good age control from independent dating techniques or the frequent occurrence of tephra layers. In total, 261 datasets for 95 of the identified lakes are available (September 2019) in public data repositories and were included in VARDA version 1.0. The datasets comprise of 70 individual chronologies from 43 lakes, 146 tephra layers from 36 lakes, 118 uncalibrated ${ }^{14} \mathrm{C}$ records from 50 lakes, and 55 varve thickness records from 23 lakes. Table 8 lists all identified lakes with their name, geographical coordinates, and available datasets, including the corresponding literature reference.
Figure 3 presents the spatial coverage of lakes and associated datasets included in VARDA 1.0. The identified lakes are located on all continents except Antarctica, with $\sim 56 \%$ located in Europe, $\sim 26 \%$ in North America, $\sim 8 \%$ in Asia, $\sim 5 \%$ in Central America and South America, $\sim 3 \%$ in Africa, and $\sim 2 \%$ in Oceania. The spatial coverage shows a distinct spatial emphasis in lake distribution on the midlatitudes of the Northern Hemisphere, especially the North Atlantic realm. In contrast, only 13 of the 190 lake archives are located on the Southern Hemisphere.

Figure 4 presents the temporal distribution of datasets included in VARDA 1.0. The combined chronologies span the entire last glacial cycle with a minimum age range of 87 years 


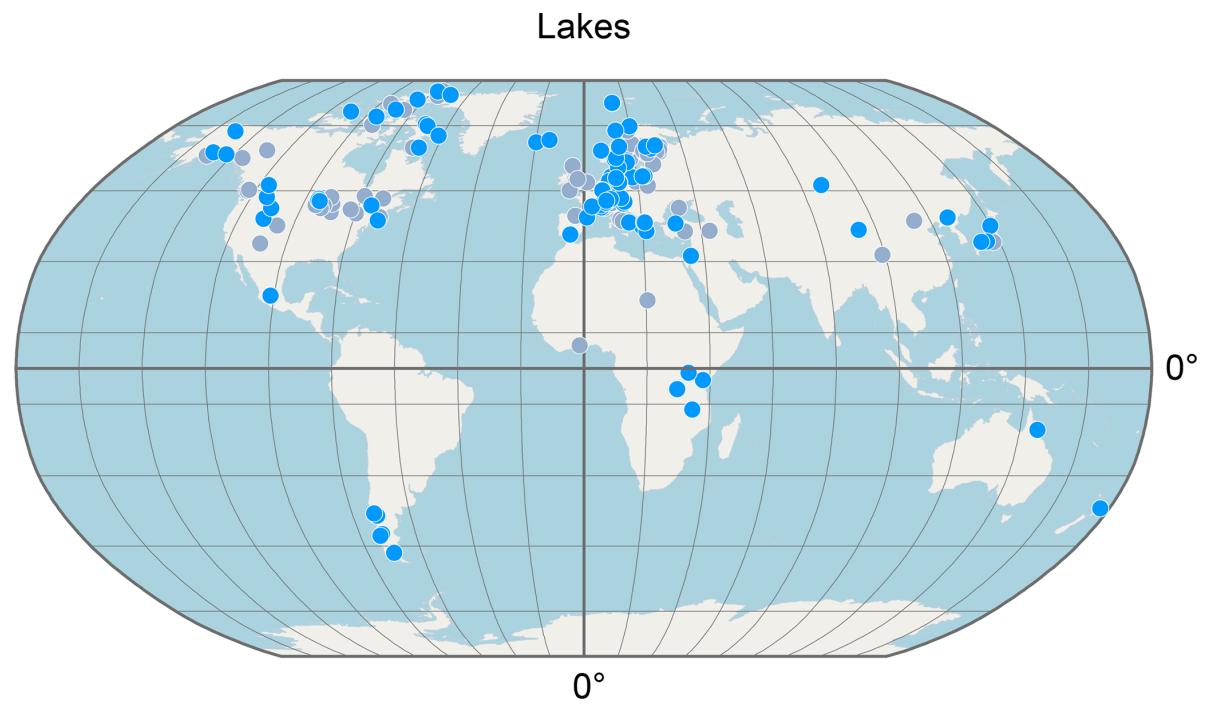

Chronologies

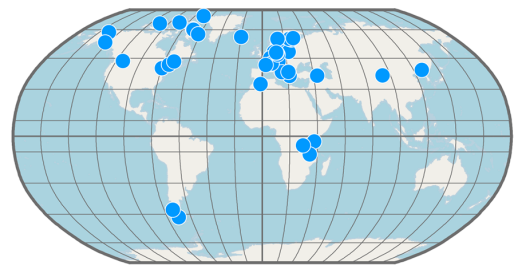

Uncalibrated ${ }^{14} \mathrm{C}$

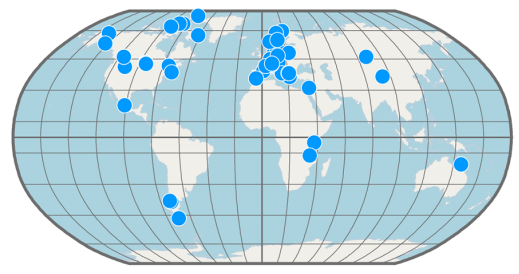

Data available
Tephra layers

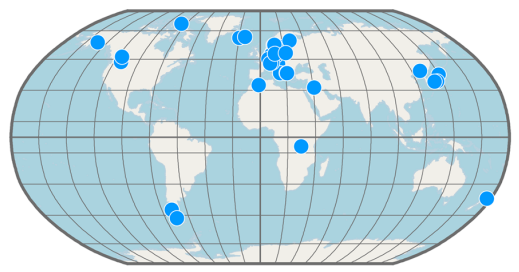

Varve thickness

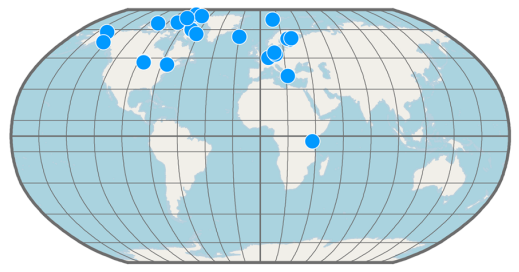

Data not available

Figure 3. Spatial distribution of identified lakes and collected datasets included in VARDA 1.0. Data availability is indicated by blue-coloured dots.

(from -60 to $27 \mathrm{BP}$ ) for Lake Woserin (Czymzik et al., 2016) and a maximal age range of 1208643 years (from 10475 to 1219118 BP) for Lake Malawi (Ivory et al., 2018). However, none of the chronologies entirely covers the last glacial cycle on its own, illustrating the need to generate multisite networks to effectively cover long time periods for environmental reconstructions. For network synchronization purposes, 146 individual tephra layers reported for sediment composite profiles in 36 lakes were identified from the published literature. A total of 30 tephra layers are reported as occurring in more than one lake and are therefore suitable for synchronization.

\section{Data availability}

All datasets are available online at https://doi.org/10.5880/GFZ.4.3.2019.003 (Ramisch et al., 2019) in JavaScript Object Notation (JSON) format. The benefit of this data format is its accurate depiction of the VARDA data model, including the relationships between data nodes. Additionally, all datasets are also available in CSV format. The VARDA graph database and the user interface can be assessed online via the following URL: https://varve.gfz-potsdam.de (last access: 15 September 2020). Support for VARDA is provided under varve@gfz-potsdam.de. 


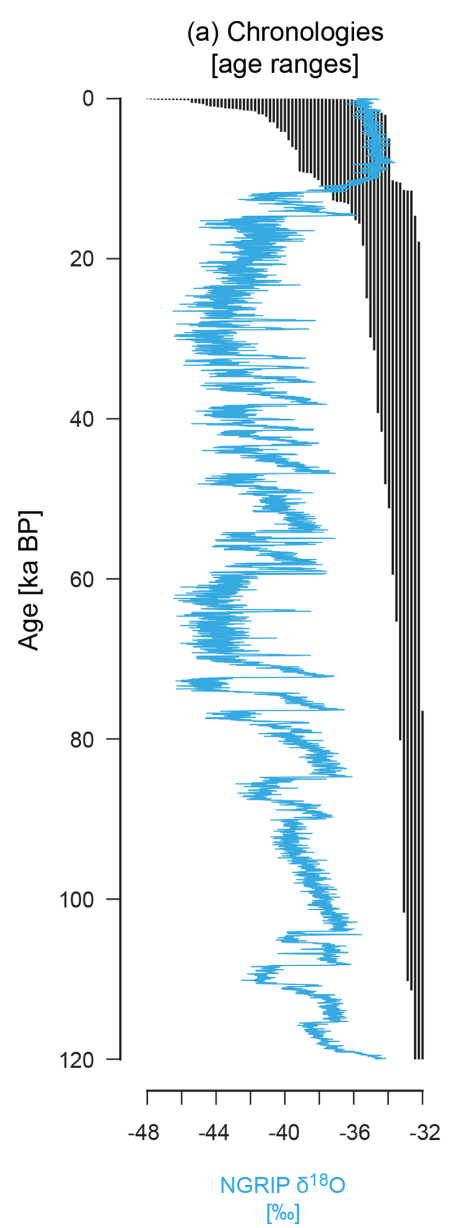

(b) Tephra layers

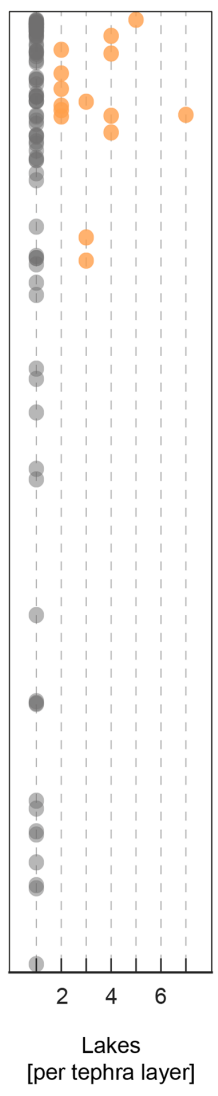

(c) Uncalibrated ${ }^{14} \mathrm{C}$
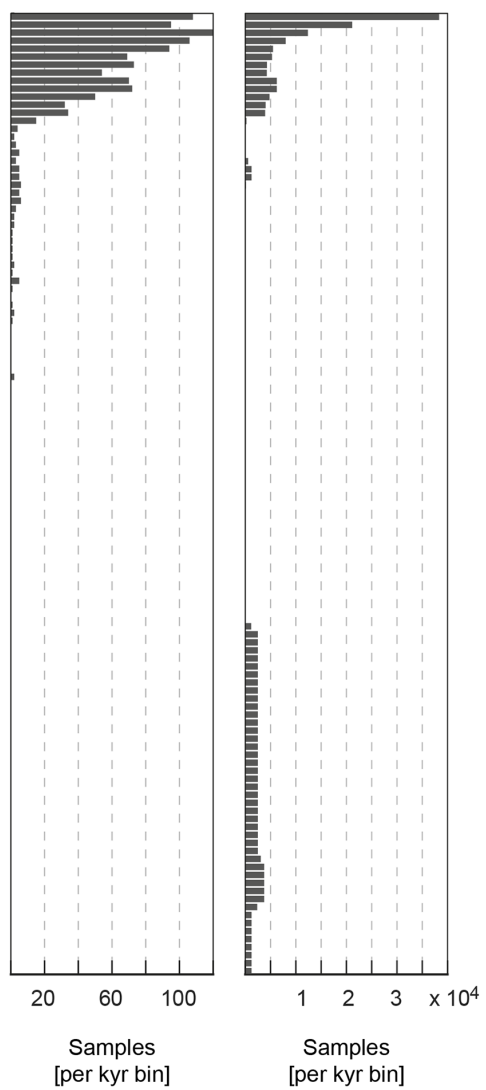

Figure 4. Temporal distribution of datasets in VARDA 1.0. (a) Age range of chronologies indicated by black bars, where each bar indicates the coverage of an individual chronology. The NGRIP stable oxygen record (Andersen et al., 2004) with the GICC05 chronology (Vinther et al., 2006; Rasmussen et al., 2006; Andersen et al., 2006; Svensson et al., 2005) is shown as a temporal reference curve. (b) Tephra layers associated with lakes included in VARDA. Dots indicate the number of lakes associated with a single tephra layer. (c) Number of samples per kiloyear bin of uncalibrated ${ }^{14} \mathrm{C}$ measurements. (d) Number of samples per kiloyear bin of individual varve thickness measurements.

\section{Conclusion and future developments}

VARDA offers a user-friendly and time-efficient way to explore the multitude of palaeoenvironmental data from varved lake archives. Due to the integration of precise chronologies and isochrones from tephra event layers into a modern graph database, VARDA offers an easy way to construct regional to global networks of palaeoenvironmental information. These multisite networks can be used e.g. to explore and analyse leads and lags of regional climate change, large scale patterns in environmental variability or differentiated proxy responses within and between archives. The first version of VARDA presented here includes all technological requirements and tools for future upgrades and developments. Presently, we are working on the integration of (1) an advanced visualization tool, (2) a user-friendly import application and (3) additional proxy data such as stable isotopes and geochemical data, as priority goals for the next update. Additionally, the source code of the database application will be made available for the public in a separate contribution. In general, VARDA is intended to be community-based effort, and we welcome and encourage the participation of varve specialists and the broader palaeoenvironmental community for the further development and application of this tool.

Author contributions. AR coordinated the manuscript writing and wrote most parts, except Sect. 3, which was written by AlB and MD. All authors contributed to manuscript writing. AlB, AR, and $\mathrm{AcB}$ carried out the data compilation and designed the standardization scheme with contributions from IN, MJB, JM, and NN for tephrochronological data; RT, JM, FO, BP, and CB for ${ }^{14} \mathrm{C}$ data and chronologies; and JM, FO, and RT for varve thickness data. AlB, $\mathrm{MD}$, and AR collected meta-information with contributions from AcB, RT, IN, JM, BP, SP, and BB for the standardization of metainformation. MD and $\mathrm{AlB}$ designed the graphical user interface for the database. MD implemented the user client and the server appli- 
cation with the help of MK. All authors reviewed the database and provided valuable feedback. AcB and AR coordinated the project.

Competing interests. The authors declare that they have no conflict of interests.

Special issue statement. This article is part of the special issue "Paleoclimate data synthesis and analysis of associated uncertainty (BG/CP/ESSD inter-journal SI)". It is not associated with a conference.

Acknowledgements. This work was supported by German Federal Ministry of Education and Research (BMBF) as a Research for Sustainability initiative (FONA; http://www.fona.de, last access: 15 September 2020) through the Palmod project. We thank Pierre Francus and an anonymous reviewer for their constructive feedback on an earlier version of the manuscript. We also like to thank Malte Räuchele, Laura Schley, Konstantin Mittelbach, Anna Beer, Helena Rollmann, Ole Tölle, Vincent Moll, and Robert Keil for their valuable support in the data compilation and graph database generation.

Financial support. This research has been supported by the Bundesministerium für Bildung und Forschung (grant no. 01LP1510A).

Review statement. This paper was edited by David Carlson and reviewed by Pierre Francus and one anonymous referee.

\section{References}

Allen, J. R. M., Brandt, U., Brauer, A., Hubberten, H.-W., Huntley, B., Keller, J., Kraml, M., Mackensen, A., Mingram, J., Negendank, J. F. W., Nowaczyk, N. R., Oberhänsli, H., Watts, W. A., Wulf, S., and Zolitschka, B.: Rapid environmental changes in southern Europe during the last glacial period, Nature, 400, 740-743, https://doi.org/10.1038/23432, 1999.

Amann, B., Szidat, S., and Grosjean, M.: A millennial-long record of warm season precipitation and flood frequency for the North-western Alps inferred from varved lake sediments: implications for the future, Quaternary Sci. Rev., 115, 89-100, https://doi.org/10.1016/j.quascirev.2015.03.002, 2015.

Andersen, K. K., Azuma, N., Barnola, J. M., Bigler, M., Biscaye, P., Caillon, N., Chappellaz, J., Clausen, H. B., Dahl-Jensen, D., Fischer, H., Flückiger, J., Fritzsche, D., Fujii, Y., Goto-Azuma, K., Grønvold, K., Gundestrup, N. S., Hansson, M., Huber, C., Hvidberg, C. S., Johnsen, S. J., Jonsell, U., Jouzel, J., Kipfstuhl, S., Landais, A., Leuenberger, M., Lorrain, R., Masson-Delmotte, V., Miller, H., Motoyama, H., Narita, H., Popp, T., Rasmussen, S. O., Raynaud, D., Rothlisberger, R., Ruth, U., Samyn, D., Schwander, J., Shoji, H., Siggard-Andersen, M. L., Steffensen, J. P., Stocker, T., Sveinbjörnsdóttir, A. E., Svensson, A., Takata, M., Tison, J. L., Thorsteinsson, T., Watanabe, O., Wilhelms, F., and White, J. W. C.: High-resolution record of Northern Hemisphere climate extending into the last interglacial period, Nature, 431 , 147-151, https://doi.org/10.1038/nature02805, 2004.

Andersen, K. K., Svensson, A., Johnsen, S. J., Rasmussen, S. O., Bigler, M., Röthlisberger, R., Ruth, U., Siggaard-Andersen, M.L., Steffensen, J. P., Dahl-Jensen, D., Vinther, B. M., and Clausen, H. B.: The Greenland Ice Core Chronology 2005, 1542 ka. Part 1: Constructing the time scale, Quaternary Sci. Rev., 25, 3246-3257, https://doi.org/10.1016/j.quascirev.2006.08.002, 2006.

Ariztegui, D., Bösch, P., and Davaud, E.: Dominant ENSO frequencies during the Little Ice Age in Northern Patagonia: The varved record of proglacial Lago Frías, Argentina, Quatern. Int., 161, 46-55, https://doi.org/10.1016/j.quaint.2006.10.022, 2007.

Atsawawaranunt, K., Comas-Bru, L., Amirnezhad Mozhdehi, S., Deininger, M., Harrison, S. P., Baker, A., Boyd, M., Kaushal, N., Ahmad, S. M., Ait Brahim, Y., Arienzo, M., Bajo, P., Braun, K., Burstyn, Y., Chawchai, S., Duan, W., Hatvani, I. G., Hu, J., Kern, Z., Labuhn, I., Lachniet, M., Lechleitner, F. A., Lorrey, A., Pérez-Mejías, C., Pickering, R., Scroxton, N., and SISAL Working Group Members: The SISAL database: a global resource to document oxygen and carbon isotope records from speleothems, Earth Syst. Sci. Data, 10, 1687-1713, https://doi.org/10.5194/essd-10-1687-2018, 2018.

Bakke, J., Dahl, S. O., Paasche, Ø., Løvlie, R., and Nesje, A.: Glacier fluctuations, equilibrium-line altitudes and palaeoclimate in Lyngen, northern Norway, during the Lateglacial and Holocene, Holocene, 15, 518-540, https://doi.org/10.1191/0959683605hl815rp, 2005.

Balascio, N. L., Zhang, Z., Bradley, R. S., Perren, B., Dahl, S. O., and Bakke, J.: A multi-proxy approach to assessing isolation basin stratigraphy from the Lofoten Islands, Norway, Quaternary Res., 75, 288-300, https://doi.org/10.1016/j.yqres.2010.08.012, 2011.

Bendle, J. M., Palmer, A. P., Thorndycraft, V. R., and Matthews, I. P.: High-resolution chronology for deglaciation of the Patagonian Ice Sheet at Lago Buenos Aires $\left(46.5^{\circ} \mathrm{S}\right)$ revealed through varve chronology and Bayesian age modelling, Quaternary Sci. Rev., 177, 314-339, https://doi.org/10.1016/j.quascirev.2017.10.013, 2017.

Berke, M. A., Johnson, T. C., Werne, J. P., Grice, K., Schouten, S., and Sinninghe Damsté, J. S.: Molecular records of climate variability and vegetation response since the Late Pleistocene in the Lake Victoria basin, East Africa, Quaternary Sci. Rev., 55, 5974, https://doi.org/10.1016/j.quascirev.2012.08.014, 2012.

Bertrand, S., Castiaux, J., and Juvigné, E.: Tephrostratigraphy of the late glacial and Holocene sediments of Puyehue Lake (Southern Volcanic Zone, Chile, $40^{\circ} \mathrm{S}$ ), Quaternary Res., 70, 343-357, https://doi.org/10.1016/j.yqres.2008.06.001, 2008.

Bird, B. W., Abbott, M. B., Finney, B. P., and Kutchko, B.: A 2000 year varve-based climate record from the central Brooks Range, Alaska, J. Paleolimnol., 41, 25-41, https://doi.org/10.1007/s10933-008-9262-y, 2008.

Blaauw, M. and Christeny, J. A.: Flexible paleoclimate age-depth models using an autoregressive gamma process, Bayesian Anal., 6, 457-474, https://doi.org/10.1214/11-BA618, 2011.

Blaauw, M., van Geel, B., Kristen, I., Plessen, B., Lyaruu, A., Engstrom, D. R., van der Plicht, J., and Verschuren, D.: Highresolution ${ }^{14} \mathrm{C}$ dating of a 25,000 -year lake-sediment record 
from equatorial East Africa, Quaternary Sci. Rev., 30, 30433059, https://doi.org/10.1016/j.quascirev.2011.07.014, 2011.

Brauer, A. and Casanova, J.: Chronology and depositional processes of the laminated sediment record from Lac d'Annecy, French Alps, J. Paleolimnol., 25, 163-177, https://doi.org/10.1023/a:1008136029735, 2001.

Brauer, A., Endres, C., Zolitschka, B., and FW Negendank, J.: AMS Radiocarbon and Varve Chronology from the Annually Laminated Sediment Record of Lake Meerfelder Maar, Germany, Radiocarbon, 42, 355-368, https://doi.org/10.1017/s0033822200030307, 2000.

Brauer, A., Hajdas, I., Blockley, S. P. E., Bronk Ramsey, C., Christl, M., Ivy-Ochs, S., Moseley, G. E., Nowaczyk, N. N., Rasmussen, S. O., Roberts, H. M., Spötl, C., Staff, R. A., and Svensson, A.: The importance of independent chronology in integrating records of past climate change for the $60-8 \mathrm{ka}$ INTIMATE time interval, Quaternary Sci. Rev., 106, 47-66, https://doi.org/10.1016/j.quascirev.2014.07.006, 2014.

Brauer, A., Haug, G. H., Dulski, P., Sigman, D. M., and Negendank, J. F. W.: An abrupt wind shift in western Europe at the onset of the Younger Dryas cold period, Nat. Geosci., 1, 520-523, https://doi.org/10.1038/ngeo263, 2008.

Bronk Ramsey, C.: Deposition models for chronological records, Quaternary Sci. Rev., 27, 42-60, 2007.

Chassiot, L., Chapron, E., Di Giovanni, C., Albéric, P., Lajeunesse, P., Lehours, A.-C., and Meybeck, M.: Extreme events in the sedimentary record of maar Lake Pavin: Implications for natural hazards assessment in the French Massif Central, Quaternary Sci. Rev., 141, 9-25, https://doi.org/10.1016/j.quascirev.2016.03.020, 2016.

Clark, J. S., Merkt, J., and Muller, H.: Post-Glacial Fire, Vegetation, and Human History on the Northern Alpine Forelands, South-Western Germany, J. Ecol., 77, 897, https://doi.org/10.2307/2260813, 1989.

Clement, A. C. and Peterson, L. C.: Mechanisms of abrupt climate change of the last glacial period, Rev. Geophys., 46, RG4002, https://doi.org/10.1029/2006RG000204, 2008.

Colman, S. M., Rosenbaum, J. G., Kaufman, D. S., Dean, W. E., and McGeehin, J. P.: Radiocarbon ages and age models for the past 30,000 years in Bear Lake, Utah and Idaho, in: Paleoenvironments of Bear Lake, Utah and Idaho, and its catchment, Geological Society of America, vol. 450, https://doi.org/10.1130/2009.2450(05), 2009.

Cook, T. L., Bradley, R. S., Stoner, J. S., and Francus, P.: Five thousand years of sediment transfer in a high arctic watershed recorded in annually laminated sediments from Lower Murray Lake, Ellesmere Island, Nunavut, Canada, J. Paleolimnol., 41, 77-94, https://doi.org/10.1007/s10933-008-9252-0, 2008.

Corella, J. P., Moreno, A., Morellón, M., Rull, V., Giralt, S., Rico, M. T., Pérez-Sanz, A., and Valero-Garcés, B. L.: Climate and human impact on a meromictic lake during the last 6,000 years (Montcortès Lake, Central Pyrenees, Spain), J. Paleolimnol., 46, 351-367, https://doi.org/10.1007/s10933-0109443-3, 2010.

Courtney Mustaphi, C. J. and Gajewski, K.: Holocene sediments from a coastal lake on northern Devon Island, Nunavut, Canada, edited by O. Lian, Can. J. Earth Sci., 50, 564-575, https://doi.org/10.1139/cjes-2012-0143, 2013.
Cuven, S., Francus, P., and Lamoureux, S.: Mid to Late Holocene hydroclimatic and geochemical records from the varved sediments of East Lake, Cape Bounty, Canadian High Arctic, Quaternary Sci. Rev., 30, 2651-2665, https://doi.org/10.1016/j.quascirev.2011.05.019, 2011.

Czymzik, M., Brauer, A., Dulski, P., Plessen, B., Naumann, R., von Grafenstein, U., and Scheffler, R.: Orbital and solar forcing of shifts in Mid- to Late Holocene flood intensity from varved sediments of pre-alpine Lake Ammersee (southern Germany), Quaternary Sci. Rev., 61, 96-110, https://doi.org/10.1016/j.quascirev.2012.11.010, 2013.

Czymzik, M., Dreibrodt, S., Feeser, I., Adolphi, F., and Brauer, A.: Mid-Holocene humid periods reconstructed from calcite varves of the Lake Woserin sediment record (north-eastern Germany), Holocene, 26, 935-946, https://doi.org/10.1177/0959683615622549, 2016.

Dean, W. E. and Megard, R. O.: Environment of deposition of CaCO3 in Elk Lake, Minnesota, in: Elk Lake, Minnesota: Evidence for Rapid Climate Change in the North-Central United States, Geological Society of America, vol. 276, 97-114, https://doi.org/10.1130/spe276-p97, 1993.

Diedrich, K. E. and Loso, M. G.: Transient impacts of Little Ice Age glacier expansion on sedimentation processes at glacier-dammed Iceberg Lake, southcentral Alaska, J. Paleolimnol., 48, 115-132, https://doi.org/10.1007/s10933-012-9614-5, 2012.

Dietze, E., Brykała, D., Schreuder, L. T., Jażdżewski, K., Blarquez, O., Brauer, A., Dietze, M., Obremska, M., Ott, F., Pieńczewska, A., Schouten, S., Hopmans, E. C., and Słowiński, M.: Human-induced fire regime shifts during 19th century industrialization: a robust fire regime reconstruction using northern Polish lake sediments, https://doi.org/10.31223/osf.io/c9z4y, 2019.

Dörfler, W., Feeser, I., van den Bogaard, C., Dreibrodt, S., Erlenkeuser, H., Kleinmann, A., Merkt, J., and Wiethold, J.: A high-quality annually laminated sequence from Lake Belau, Northern Germany: Revised chronology and its implications for palynological and tephrochronological studies, Holocene, 22, 1413-1426, https://doi.org/10.1177/0959683612449756, 2012.

Dräger, N., Theuerkauf, M., Szeroczyńska, K., Wulf, S., Tjallingii, R., Plessen, B., Kienel, U., and Brauer, A.: Varve microfacies and varve preservation record of climate change and human impact for the last 6000 years at Lake Tiefer See (NE Germany), Holocene, 27, 450-464, https://doi.org/10.1177/0959683616660173, 2016.

Eden, D. N. and Page, M. J.: Palaeoclimatic implications of a storm erosion record from late Holocene lake sediments, North Island, New Zealand, Palaeogeogr. Palaeocl., 139, 37-58, https://doi.org/10.1016/s0031-0182(97)00136-3, 1998.

Elbert, J., Jacques-Coper, M., Van Daele, M., Urrutia, R., and Grosjean, M.: A 600 years warm-season temperature record from varved sediments of Lago Plomo, Northern Patagonia, Chile $\left(47^{\circ} \mathrm{S}\right)$, Quatern. Int., 377, 28-37, https://doi.org/10.1016/j.quaint.2015.01.004, 2015.

Engels, S., van Geel, B., Buddelmeijer, N., and Brauer, A.: High-resolution palynological evidence for vegetation response to the Laacher See eruption from the varved record of Meerfelder Maar (Germany) and other central European records, Rev. Palaeobot. Palyno., 221, 160-170, https://doi.org/10.1016/j.revpalbo.2015.06.010, 2015. 
Enters, D., Kirilova, E., Lotter, A. F., Lücke, A., Parplies, J., Jahns, S., Kuhn, G., and Zolitschka, B.: Climate change and human impact at Sacrower See (NE Germany) during the past 13,000 years: a geochemical record, J. Paleolimnol., 43, 719737, https://doi.org/10.1007/s10933-009-9362-3, 2009.

Euler, L.: Solutio problematis ad geometriam situs pertinentis, Commentarii academiae scientiarum Petropolitanae, Commentarii academiae scientiarum Petropolitanae, Vol. 8, 128-140, 1741.

Fortin, D., Praet, N., McKay, N. P., Kaufman, D. S., Jensen, B. J. L., Haeussler, P. J., Buchanan, C., and De Batist, M.: New approach to assessing age uncertainties - The 2300-year varve chronology from Eklutna Lake, Alaska (USA), Quaternary Sci. Rev., 203, 90-101, https://doi.org/10.1016/j.quascirev.2018.10.018, 2019.

Francke, A., Wagner, B., Just, J., Leicher, N., Gromig, R., Baumgarten, H., Vogel, H., Lacey, J. H., Sadori, L., Wonik, T., Leng, M. J., Zanchetta, G., Sulpizio, R., and Giaccio, B.: Sedimentological processes and environmental variability at Lake Ohrid (Macedonia, Albania) between $637 \mathrm{ka}$ and the present, Biogeosciences, 13, 1179-1196, https://doi.org/10.5194/bg-131179-2016, 2016.

Francus, P., Bradley, R. S., Abbott, M. B., Patridge, W., and Keimig, F.: Paleoclimate studies of minerogenic sediments using annually resolved textural parameters, Geophys. Res. Lett., 29, 59-1-59-4, https://doi.org/10.1029/2002g1015082, 2002.

Franke, J. G., Werner, J. P., and Donner, R. V.: Reconstructing Late Holocene North Atlantic atmospheric circulation changes using functional paleoclimate networks, Clim. Past, 13, 1593-1608, https://doi.org/10.5194/cp-13-1593-2017, 2017.

Fyfe, R. M., de Beaulieu, J. L., Binney, H., Bradshaw, R. H. W., Brewer, S., Le Flao, A., Finsinger, W., Gaillard, M. J., Giesecke, T., Gil-Romera, G., Grimm, E. C., Huntley, B., Kunes, P., Kühl, N., Leydet, M., Lotter, A. F., Tarasov, P. E., and Tonkov, S.: The European Pollen Database: Past efforts and current activities, Veg. Hist. Archaeobot., 18, 417-424, https://doi.org/10.1007/s00334-009-0215-9, 2009.

Garbe-Schönberg, C.-D., Wiethold, J., Butenhoff, D., Utech, C., and Stoffers, P.: Geochemical and palynological record in annually laminated sediments from Lake Belau (Schleswig-Holstein) reflecting paleoecology and human impact over 9000 a. Meyniana, 50, 47-70, https://doi.org/10.2312/meyniana.1998.50.47, 1998.

Gierga, M., Hajdas, I., van Raden, U. J., Gilli, A., Wacker, L., Sturm, M., Bernasconi, S. M., and Smittenberg, R. H.: Longstored soil carbon released by prehistoric land use: Evidence from compound-specific radiocarbon analysis on Soppensee lake sediments, Quaternary Sci. Rev., 144, 123-131, https://doi.org/10.1016/j.quascirev.2016.05.011, 2016.

Giguet-Covex, C., Arnaud, F., Poulenard, J., Disnar, J.-R., Delhon, C., Francus, P., David, F., Enters, D., Rey, P.-J., and Delannoy, J.-J.: Changes in erosion patterns during the Holocene in a currently treeless subalpine catchment inferred from lake sediment geochemistry (Lake Anterne, $2063 \mathrm{~m}$ a.s.1., NW French Alps): The role of climate and human activities, Holocene, 21, 651-665, https://doi.org/10.1177/0959683610391320, 2011.

Grafenstein, U. v.: A Mid-European Decadal Isotope-Climate Record from 15,500 to 5000 Years B.P., Science, 284, 16541657, https://doi.org/10.1126/science.284.5420.1654, 1999.

Guyard, H., Chapron, E., St-Onge, G., Anselmetti, F. S., Arnaud, F., Magand, O., Francus, P., and Mélières, M.-A.: High-altitude varve records of abrupt environmental changes and mining activity over the last 4000 years in the Western French Alps (Lake Bramant, Grandes Rousses Massif), Quaternary Sci. Rev., 26, 2644-2660, https://doi.org/10.1016/j.quascirev.2007.07.007, 2007.

Haberzettl, T., Corbella, H., Fey, M., Janssen, S., Lücke, A., Mayr, C., Ohlendorf, C., Schäbitz, F., Schleser, G. H., Wille, M., Wulf, S., and Zolitschka, B.: Lateglacial and Holocene wet - dry cycles in southern Patagonia: chronology, sedimentology and geochemistry of a lacustrine record from Laguna Potrok Aike, Argentina, Holocene, 17, 297-310, https://doi.org/10.1177/0959683607076437, 2007.

Haenssler, E., Nadeau, M.-J., Vött, A., and Unkel, I.: Natural and human induced environmental changes preserved in a Holocene sediment sequence from the Etoliko Lagoon, Greece: New evidence from geochemical proxies, Quatern. Int., 308-309, 89104, https://doi.org/10.1016/j.quaint.2012.06.031, 2013.

Hajdas, I. and Michczyński, A.: Age-Depth Model of Lake Soppensee (Switzerland) Based on the High-Resolution ${ }^{14} \mathrm{C}$ Chronology Compared with Varve Chronology, Radiocarbon, 52, 1027-1040, https://doi.org/10.1017/s0033822200046117, 2010.

Hajdas, I., Bonani, G., Moreno, P. I., and Ariztegui, D.: Precise radiocarbon dating of Late-Glacial cooling in mid-latitude South America, Quaternary Res., 59, 70-78, https://doi.org/10.1016/s0033-5894(02)00017-0, 2003.

Hajdas, I., Bonani, G., Zolitschka, B., Brauer, A., and Negendank, J.: ${ }^{14} \mathrm{C}$ Ages of Terrestrial Macrofossils from Lago Grande Di Monticchio (Italy), Radiocarbon, 40, 803-807, https://doi.org/10.1017/s0033822200018750, 1997.

Haltiahovi, E., Saarinen, T., and Kukkonen, M.: A 2000year record of solar forcing on varved lake sediment in eastern Finland, Quaternary Sci. Rev., 26, 678-689, https://doi.org/10.1016/j.quascirev.2006.11.005, 2007.

Head, M. J., Taylor, L. J., and Walker, D.: ANU Radiocarbon Date List XI: Radiocarbon Dates from Lakes Barrine and Eacham, Atherton Tableland, North Queensland, Australia, Radiocarbon, 36, 73-94, https://doi.org/10.1017/s003382220001434x, 1994.

Hu, F. S., Slawinski, D., Wright, H. E., Ito, E., Johnson, R. G., Kelts, K. R., McEwan, R. F., and Boedigheimer, A.: Abrupt changes in North American climate during early Holocene times, Nature, 400, 437-440, https://doi.org/10.1038/22728, 1999.

Hubeny, J. B., King, J. W., and Cantwell, M.: Anthropogenic influences on estuarine sedimentation and ecology: examples from the varved sediments of the Pettaquamscutt River Estuary, Rhode Island, J. Paleolimnol., 41, 297-314, https://doi.org/10.1007/s10933-008-9226-2, 2008.

Huntley, B., Watts, W. A., Allen, J. R. M., and Zolitschka, B.: Palaeoclimate, chronology and vegetation history of the Weichselian Lateglacial: comparative analysis of data from three cores at Lago Grande di Monticchio, southern Italy, Quaternary Sci. Rev., 18, 945-960, https://doi.org/10.1016/s02773791(99)00007-4, 1999.

Ivory, S. J., Blome, M. W., King, J. W., McGlue, M. M., Cole, J. E., and Cohen, A. S.: Environmental change explains cichlid adaptive radiation at Lake Malawi over the past 1.2 million years, P. Natl. Acad. Sci. USA, 113, 11895-11900, https://doi.org/10.1073/pnas.1611028113, 2016.

Jones, G., Lane, C. S., Brauer, A., Davies, S. M., de Bruijn, R., Engels, S., Haliuc, A., Hoek, W. Z., Merkt, J., Sachse, D., 
Turner, F., and Wagner-Cremer, F.: The Lateglacial to early Holocene tephrochronological record from Lake Hämelsee, Germany: a key site within the European tephra framework, Boreas, 47, 28-40, https://doi.org/10.1111/bor.12250, 2017.

Jonkers, L., Cartapanis, O., Langner, M., McKay, N., Mulitza, S., Strack, A., and Kucera, M.: Integrating palaeoclimate time series with rich metadata for uncertainty modelling: strategy and documentation of the PalMod 130k marine palaeoclimate data synthesis, Earth Syst. Sci. Data, 12, 1053-1081, https://doi.org/10.5194/essd-12-1053-2020, 2020.

Kalliokoski, M., Wastegård, S., and Saarinen, T.: Rhyolitic and dacitic component of the Askja 1875 tephra in southern and central Finland: first step towards a Finnish tephrochronology, J. Quaternary Sci., 34, 29-39, https://doi.org/10.1002/jqs.3078, 2018.

Kato, M., Fukusawa, H., and Yasuda, Y.: Varved lacustrine sediments of Lake Tougou-ike, Western Japan, with reference to Holocene sea-level changes in Japan, Quatern. Int., 105, 33-37, https://doi.org/10.1016/s1040-6182(02)00148-9, 2003.

Kliem, P., Enters, D., Hahn, A., Ohlendorf, C., Lisé-Pronovost, A., St-Onge, G., Wastegård, S., and Zolitschka, B.: Lithology, radiocarbon chronology and sedimentological interpretation of the lacustrine record from Laguna Potrok Aike, southern Patagonia, Quaternary Sci. Rev., 71, 54-69, https://doi.org/10.1016/j.quascirev.2012.07.019, 2013.

Koutsodendris, A., Brauer, A., Reed, J. M., Plessen, B., Friedrich, O., Hennrich, B., Zacharias, I., and Pross, J.: Climate variability in SE Europe since 1450 AD based on a varved sediment record from Etoliko Lagoon (Western Greece), Quaternary Sci. Rev., 159, 63-76, https://doi.org/10.1016/j.quascirev.2017.01.010, 2017.

Labuhn, I., Hammarlund, D., Chapron, E., Czymzik, M., Dumoulin, J.-P., Nilsson, A., Régnier, E., Robygd, J., and von Grafenstein, U.: Holocene Hydroclimate Variability in Central Scandinavia Inferred from Flood Layers in Contourite Drift Deposits in Lake Storsjön, Quaternary, 1, 2, https://doi.org/10.3390/quat1010002, 2018.

Lafontaine-Boyer, K. and Gajewski, K.: Vegetation dynamics in relation to late Holocene climate variability and disturbance, Outaouais, Québec, Canada, Holocene, 24, 1515-1526, https://doi.org/10.1177/0959683614544054, 2014.

Lamoureux, S. and Bradley, R.: A late Holocene varved sediment record of environmental change from northern Ellesmere Island, Canada, J. Paleolimnol., 16, 239-255, https://doi.org/10.1007/bf00176939, 1996.

Lane, C. S., Andrič, M., Cullen, V. L., and Blockley, S. P. E.: The occurrence of distal Icelandic and Italian tephra in the Lateglacial of Lake Bled, Slovenia, Quaternary Sci. Rev., 30, 1013-1018, https://doi.org/10.1016/j.quascirev.2011.02.014, 2011.

Lane, C. S., Brauer, A., Blockley, S. P. E., and Dulski, P.: Volcanic ash reveals time-transgressive abrupt climate change during the Younger Dryas, Geology, 41, 1251-1254, https://doi.org/10.1130/G34867.1, 2013.

Lane, C. S., Brauer, A., Martín-Puertas, C., Blockley, S. P. E., Smith, V. C., and Tomlinson, E. L.: The Late Quaternary tephrostratigraphy of annually laminated sediments from Meerfelder Maar, Germany, Quaternary Sci. Rev., 122, 192-206, https://doi.org/10.1016/j.quascirev.2015.05.025, 2015.
Lane, C. S., Martin-Jones, C. M., and Johnson, T. C.: A cryptotephra record from the Lake Victoria sediment core record of Holocene palaeoenvironmental change, Holocene, 28, 19091917, https://doi.org/10.1177/0959683618798163, 2018.

Larsen, D. J., Miller, G. H., and Geirsdóttir, Á.: Asynchronous Little Ice Age glacier fluctuations in Iceland and European Alps linked to shifts in subpolar North Atlantic circulation, Earth Planet. Sc. Lett., 380, 52-59, https://doi.org/10.1016/j.epsl.2013.07.028, 2013.

Larsen, D. J., Miller, G. H., Geirsdóttir, Á., and Thordarson, T.: A 3000-year varved record of glacier activity and climate change from the proglacial lake Hvítárvatn, Iceland, Quaternary Sci. Rev., 30, 2715-2731, https://doi.org/10.1016/j.quascirev.2011.05.026, 2011.

Latif, M., Claussen, M., Schulz, M., and Brüchner, T.: Comprehensive Earth system models of the last glacial cycle, EOS, 97, https://doi.org/10.1029/2016EO059587, 2016.

Lauterbach, S., Brauer, A., Andersen, N., Danielopol, D. L., Dulski, P., Hüls, M., Milecka, K., Namiotko, T., Plessen, B., Grafenstein, U. V., and Participants, D.: Multi-proxy evidence for early to mid-Holocene environmental and climatic changes in northeastern Poland, Boreas, 40, 57-72, https://doi.org/10.1111/j.1502-3885.2010.00159.x, 2010.

Lauterbach, S., Brauer, A., Andersen, N., Danielopol, D. L., Dulski, P., Hüls, M., Milecka, K., Namiotko, T., Obremska, M., and Von Grafenstein, U.: Environmental responses to Lateglacial climatic fluctuations recorded in the sediments of pre-Alpine Lake Mondsee (northeastern Alps), J. Quaternary Sci., 26, 253-267, https://doi.org/10.1002/jqs.1448, 2011.

Leicher, N., Zanchetta, G., Sulpizio, R., Giaccio, B., Wagner, B., Nomade, S., Francke, A., and Del Carlo, P.: First tephrostratigraphic results of the DEEP site record from Lake Ohrid (Macedonia and Albania), Biogeosciences, 13, 2151-2178, https://doi.org/10.5194/bg-13-2151-2016, 2016.

Leonard, E. M. and Reasoner, M. A.: A Continuous Holocene Glacial Record Inferred from Proglacial Lake Sediments in Banff National Park, Alberta, Canada, Quaternary Res., 51, 1-13, https://doi.org/10.1006/qres.1998.2009, 1999.

Litt, T., Schölzel, C., Kühl, N., and Brauer, A.: Vegetation and climate history in the Westeifel Volcanic Field (Germany) during the past 11000 years based on annually laminated lacustrine maar sediments, Boreas, 38, 679-690, https://doi.org/10.1111/j.1502-3885.2009.00096.x, 2009.

Loso, M. G.: Summer temperatures during the Medieval Warm Period and Little Ice Age inferred from varved proglacial lake sediments in southern Alaska, J. Paleolimnol., 41, 117-128, https://doi.org/10.1007/s10933-008-9264-9, 2008.

Macleod, A., Brunnberg, L., Wastegård, S., Hang, T., and Matthews, I. P.: Lateglacial cryptotephra detected within clay varves in Östergötland, south-east Sweden, J. Quaternary Sci., 29, 605-609, https://doi.org/10.1002/jqs.2738, 2014.

Martín-Puertas, C., Valero-Garcés, B. L., Pilar Mata, M., GonzálezSampériz, P., Bao, R., Moreno, A., and Stefanova, V.: Arid and humid phases in southern Spain during the last 4000 years: the Zoñar Lake record, Córdoba, Holocene, 18, 907-921, https://doi.org/10.1177/0959683608093533, 2008.

Martin-Puertas, C., Brauer, A., Dulski, P., and Brademann, B.: Testing climate-proxy stationarity throughout the Holocene: an example from the varved sediments of Lake Meer- 
felder Maar (Germany), Quaternary Sci. Rev., 58, 56-65, https://doi.org/10.1016/j.quascirev.2012.10.023, 2012.

Martin-Puertas, C., Brauer, A., Wulf, S., Ott, F., Lauterbach, S., and Dulski, P.: Annual proxy data from Lago Grande di Monticchio (southern Italy) between 76 and $112 \mathrm{ka}$ : new chronological constraints and insights on abrupt climatic oscillations, Clim. Past, 10, 2099-2114, https://doi.org/10.5194/cp-10-2099-2014, 2014.

Mellström, A., Muscheler, R., Snowball, I., Ning, W., and Haltia, E.: Radiocarbon Wiggle-Match Dating of Bulk Sediments - How Accurate can It Be?, Radiocarbon, 55, 1173-1186, https://doi.org/10.1017/s0033822200048086, 2013.

Migowski, C., Agnon, A., Bookman, R., Negendank, J. F., and Stein, M.: Recurrence pattern of Holocene earthquakes along the Dead Sea transform revealed by varve-counting and radiocarbon dating of lacustrine sediments, Earth Planet. Sc. Lett., 222, 301314, https://doi.org/10.1016/j.eps1.2004.02.015, 2004.

Millard, A. R.: Conventions for Reporting Radiocarbon Determinations, Radiocarbon, 56, 555-559, https://doi.org/10.2458/56.17455, 2014.

Mingram, J., Stebich, M., Schettler, G., Hu, Y., Rioual, P., Nowaczyk, N., Dulski, P., You, H., Opitz, S., Liu, Q., and Liu, J.: Millennial-scale East Asian monsoon variability of the last glacial deduced from annually laminated sediments from Lake Sihailongwan, N. E. China, Quaternary Sci. Rev., 201, 5776, https://doi.org/10.1016/j.quascirev.2018.09.023, 2018.

Moore, J. J., Hughen, K. A., Miller, G. H., and Overpeck, J. T.: Little Ice Age recorded in summer temperature reconstruction from vared sediments of Donard Lake, Baffin Island, Canada, J. Paleolimnol., 25, 503-517, https://doi.org/10.1023/a:1011181301514, 2001.

Morellón, M., Anselmetti, F. S., Ariztegui, D., Brushulli, B., Sinopoli, G., Wagner, B., Sadori, L., Gilli, A., and Pambuku, A.: Human-climate interactions in the central Mediterranean region during the last millennia: The laminated record of Lake Butrint (Albania), Quaternary Sci. Rev., 136, 134-152, https://doi.org/10.1016/j.quascirev.2015.10.043, 2016.

Neugebauer, I., Brauer, A., Dräger, N., Dulski, P., Wulf, S., Plessen, B., Mingram, J., Herzschuh, U., and Brande, A.: A Younger Dryas varve chronology from the Rehwiese palaeolake record in NE-Germany, Quaternary Sci. Rev., 36, 91-102, https://doi.org/10.1016/j.quascirev.2011.12.010, 2012.

Neugebauer, I., Brauer, A., Schwab, M. J., Dulski, P., Frank, U., Hadzhiivanova, E., Kitagawa, H., Litt, T., Schiebel, V., Taha, N., and Waldmann, N. D.: Evidences for centennial dry periods at $\sim 3300$ and $\sim 2800$ cal. yr BP from micro-facies analyses of the Dead Sea sediments, Holocene, 25, 1358-1371, https://doi.org/10.1177/0959683615584208, 2015.

O’Beirne, M. D., Werne, J. P., Hecky, R. E., Johnson, T. C., Katsev, S., and Reavie, E. D.: Anthropogenic climate change has altered primary productivity in Lake Superior, Nat. Commun., 8, 15713, https://doi.org/10.1038/ncomms15713, 2017.

Ott, F., Kramkowski, M., Wulf, S., Plessen, B., Serb, J., Tjallingii, R., Schwab, M., Słowiński, M., Brykała, D., Tyszkowski, S., Putyrskaya, V., Appelt, O., Błaszkiewicz, M., and Brauer, A.: Site-specific sediment responses to climate change during the last 140 years in three varved lakes in Northern Poland, Holocene, 28, 464-477, https://doi.org/10.1177/0959683617729448, 2017.
PAGES2k Consortium, Emile-Geay, J., McKay, N. P., Kaufman, D. S., von Gunten, L., Wang, J., Anchukaitis, K. J., Abram, N. J., Addison, J. A., Curran, M. A. J., Evans, M. N., Henley, B. J., Hao, Z., Martrat, B., McGregor, H. V, Neukom, R., Pederson, G. T., Stenni, B., Thirumalai, K., Werner, J. P., Xu, C., Divine, D. V, Dixon, B. C., Gergis, J., Mundo, I. A., Nakatsuka, T., Phipps, S. J., Routson, C. C., Steig, E. J., Tierney, J. E., Tyler, J. J., Allen, K. J., Bertler, N. A. N., Björklund, J., Chase, B. M., Chen, M.-T., Cook, E., de Jong, R., DeLong, K. L., Dixon, D. A., Ekaykin, A. A., Ersek, V., Filipsson, H. L., Francus, P., Freund, M. B., Frezzotti, M., Gaire, N. P., Gajewski, K., Ge, Q., Goosse, H., Gornostaeva, A., Grosjean, M., Horiuchi, K., Hormes, A., Husum, K., Isaksson, E., Kandasamy, S., Kawamura, K., Kilbourne, K. H., Koç, N., Leduc, G., Linderholm, H. W., Lorrey, A. M., Mikhalenko, V., Mortyn, P. G., Motoyama, H., Moy, A. D., Mulvaney, R., Munz, P. M., Nash, D. J., Oerter, H., Opel, T., Orsi, A. J., Ovchinnikov, D. V, Porter, T. J., Roop, H. A., Saenger, C., Sano, M., Sauchyn, D., Saunders, K. M., Seidenkrantz, M.-S., Severi, M., Shao, X., Sicre, M.-A., Sigl, M., Sinclair, K., St. George, S., St. Jacques, J.-M., Thamban, M., Kuwar Thapa, U., Thomas, E. R., Turney, C., Uemura, R., Viau, A. E., Vladimirova, D. O., Wahl, E. R., White, J. W. C., Yu, Z., and Zinke, J.: A global multiproxy database for temperature reconstructions of the Common Era, Sci. Data, 4, 170088, https://doi.org/10.1038/sdata.2017.88, 2017.

Park, J., Byrne, R., Böhnel, H., Garza, R. M., and Conserva, M.: Holocene climate change and human impact, central Mexico: a record based on maar lake pollen and sediment chemistry, Quaternary Sci. Rev., 29, 618-632, https://doi.org/10.1016/j.quascirev.2009.10.017, 2010.

Paull, T. M., Finkelstein, S. A., and Gajewski, K.: Interactions between climate and landscape drive Holocene ecological change in a High Arctic lake on Somerset Island, Nunavut, Canada, Arct. Sci., 3, 17-38, https://doi.org/10.1139/as-2016-0013, 2017.

Pickarski, N., Kwiecien, O., Langgut, D., and Litt, T.: Abrupt climate and vegetation variability of eastern Anatolia during the last glacial, Clim. Past, 11, 1491-1505, https://doi.org/10.5194/cp11-1491-2015, 2015.

Pilskaln, C. H. and Johnson, T. C.: Seasonal signals in Lake Malawi sediments, Limnol. Oceanogr., 36, 544-557, https://doi.org/10.4319/lo.1991.36.3.0544, 1991.

Prasad, S. and Baier, J.: Tracking the impact of mid- to late Holocene climate change and anthropogenic activities on Lake Holzmaar using an updated Holocene chronology, Global Planet. Change, 122, 251-264, https://doi.org/10.1016/j.gloplacha.2014.08.020, 2014.

Rach, O., Brauer, A., Wilkes, H., and Sachse, D.: Delayed hydrological response to Greenland cooling at the onset of the Younger Dryas in western Europe, Nat. Geosci., 7, 109-112, https://doi.org/10.1038/ngeo2053, 2014.

Ramisch, A., Tjallingii, R., Hartmann, K., Diekmann, B., and Brauer, A.: Echo of the Younger Dryas in Holocene lake sediments on the Tibetan Plateau, Geophys. Res. Lett., 45, 11154 11163, https://doi.org/10.1029/2018GL080225, 2018.

Ramisch, A., Brauser, A., Dorn, M., Brauer, A., Blanchet, C., Brademann, B., Köppl, M., Mingram, J., Neugebauer, I., Nowaczyk, N., Ott, F., Pinkerneil, S., Plessen, B., Schwab, M. J., Tjallingii, R.: Data inventory of the varve database (VARDA): Sediment profiles, chronologies, radiocarbon dates, tephra lay- 
ers and varve thickness data, V. 1.1., GFZ Data Services, https://doi.org/10.5880/GFZ.4.3.2019.003, 2019.

Rasmussen, S. O., Andersen, K. K., Svensson, A. M., Steffensen, J. P., Vinther, B. M., Clausen, H. B., SiggaardAndersen, M.-L., Johnsen, S. J., Larsen, L. B., Dahl-Jensen, D., Bigler, M., Röthlisberger, R., Fischer, H., Goto-Azuma, K., Hansson, M. E., and Ruth, M. E.: A new Greenland ice core chronology for the last glacial termination, J. Geophys. Res., 111, D06102, https://doi.org/10.1029/2005JD006079, 2006.

Reimer, P. J., Baillie, M. G. L., Bard, E., Bayliss, A., Beck, J. W., Blackwell, P. G., Ramsey, C. B., Buck, C. E., Burr, G. S., Edwards, R. L., Friedrich, M., Grootes, P. M., Guilderson, T. P., Hajdas, I., Heaton, T. J., Hogg, A. G., Hughen, K. A., Kaiser, K. F., Kromer, B., McCormac, F. G., Manning, S. W., Reimer, R. W., Richards, D. A., Southon, J. R., Talamo, S., Turney, C. S. M., van der Plicht, J., and Weyhenmeyer, C. E.: IntCal09 and Marine09 radiocarbon age calibration curves, 0-50,000 years CAL BP, Radiocarbon, 51, 1111-1150, https://doi.org/10.1017/S0033822200034202, 2009.

Reimer, P. J., Baillie, M. G. L., Bard, E., Bayliss, A., Warren Beck, J., Bertrand, C. J. H., Blackwell, P. G., Buck, C. E., Burr, G. S., Cutler, K. B., Damon, P. E., Lawrence Edwards, R., Fairbanks, R. G., Friedrich, M., Guilderson, T. P., Hogg, A. G., Hughen, K. A., Kromer, B., McCormac, G., Manning, S., Ramsey, C. B., Reimer, R. W., Remmele, S., Southon, J. R., Stuiver, M., Talamo, S., Taylor, F. W., van der Plicht, J., and Weyhenmeyer, C. E.: IntCal04 terrestrial radiocarbon age calibration, 0-26 cal kyr BP, Radiocarbon, 46, 10291058, https://doi.org/10.1017/S0033822200032999, 2004.

Reimer, P. J., Edouard Bard, B., Alex Bayliss, B., Warren Beck, B. J., Paul Blackwell, B. G., and Christopher Bronk Ramsey, B.: Intcal13 and Marine13 Radiocarbon Age Calibration Curves 0-50,000 Years Cal BP, Radiocarbon, 55, 1869-1887, https://doi.org/10.2458/azu_js_rc.55.16947, 2013.

Roberts, N., Allcock, S. L., Arnaud, F., Dean, J. R., Eastwood, W. J., Jones, M. D., Leng, M. J., Metcalfe, S. E., Malet, E., Woodbridge, J., and Yiğitbaşıŏlu, H.: A tale of two lakes: a multiproxy comparison of Lateglacial and Holocene environmental change in Cappadocia, Turkey, J. Quaternary Sci., 31, 348-362, https://doi.org/10.1002/jqs.2852, 2016.

Rudaya, N., Nazarova, L., Novenko, E., Andreev, A., Kalugin, I., Daryin, A., Babich, V., Li, H.-C., and Shilov, P.: Quantitative reconstructions of mid- to late holocene climate and vegetation in the north-eastern altai mountains recorded in lake teletskoye, Global Planet. Change, 141, 12-24, https://doi.org/10.1016/j.gloplacha.2016.04.002, 2016.

Saarni, S., Saarinen, T., and Lensu, A.: Organic lacustrine sediment varves as indicators of past precipitation changes: a 3,000-year climate record from Central Finland, J. Paleolimnol., 53, 401413, https://doi.org/10.1007/s10933-015-9832-8, 2015a.

Saarni, S., Saarinen, T., and Dulski, P.: Between the North Atlantic Oscillation and the Siberian High: A 4000year snow accumulation history inferred from varved lake sediments in Finland, Holocene, 26, 423-431, https://doi.org/10.1177/0959683615609747, 2015 b.

Sánchez GoÑi, M. F., Desprat, S., Daniau, A.-L., Bassinot, F. C., Polanco-Martínez, J. M., Harrison, S. P., Allen, J. R. M., Anderson, R. S., Behling, H., Bonnefille, R., Burjachs, F., Carrión, J. S., Cheddadi, R., Clark, J. S., Combourieu-Nebout, N., Mustaphi,
Colin. J. Courtney, Debusk, G. H., Dupont, L. M., Finch, J. M., Fletcher, W. J., Giardini, M., González, C., Gosling, W. D., Grigg, L. D., Grimm, E. C., Hayashi, R., Helmens, K., Heusser, L. E., Hill, T., Hope, G., Huntley, B., Igarashi, Y., Irino, T., Jacobs, B., JimÉnez-Moreno, G., Kawai, S., Kershaw, A. P., Kumon, F., Lawson, I. T., Ledru, M.-P., LÉzine, A.-M., Liew, P. M., Magri, D., Marchant, R., Margari, V., Mayle, F. E., McKenzie, G. M., Moss, P., MÜller, S., MÜller, U. C., Naughton, F., Newnham, R. M., Oba, T., PÉrez-Obiol, R., Pini, R., Ravazzi, C., Roucoux, K. H., Rucina, S. M., Scott, L., Takahara, H., Tzedakis, P. C., Urrego, D. H., van Geel, B., Valencia, B. G., Vandergoes, M. J., Vincens, A., Whitlock, C. L., Willard, D. A., and Yamamoto, M.: The ACER pollen and charcoal database: a global resource to document vegetation and fire response to abrupt climate changes during the last glacial period, Earth Syst. Sci. Data, 9, 679-695, https://doi.org/10.5194/essd-9-679-2017, 2017.

Schmidt, R., van den Bogaard, C., Merkt, J., and Müller, J.: A new Lateglacial chronostratigraphic tephra marker for the southeastern Alps: The Neapolitan Yellow Tuff (NYT) in Längsee (Austria) in the context of a regional biostratigraphy and palaeoclimate, Quatern. Int., 88, 45-56, https://doi.org/10.1016/s10406182(01)00072-6, 2002.

Sheng $\mathrm{Hu}, \mathrm{F}$., Wright, H. E., Ito, E., and Lease, K.: Climatic effects of glacial Lake Agassiz in the midwestern United States during the last deglaciation, Geology, 25, 207, https://doi.org/10.1130/00917613(1997)025<0207:ceogla>2.3.co;2, 1997.

Shuman, B., Henderson, A. K., Colman, S. M., Stone, J. R., Fritz, S. C., Stevens, L. R., Power, M. J., and Whitlock, C.: Holocene lake-level trends in the Rocky Mountains, U.S.A., Quaternary Sci. Rev., 28, 1861-1879, https://doi.org/10.1016/j.quascirev.2009.03.003, 2009.

Smith, A. J., Donovan, J. J., Ito, E., and Engstrom, D. R.: Ground-water processes controlling a prairie lake's response to middle Holocene drought, Geology, 25, 391, https://doi.org/10.1130/00917613(1997)025<0391:gwpcap>2.3.co;2, 1997.

Smith, V. C., Staff, R. A., Blockley, S. P. E., Bronk Ramsey, C., Nakagawa, T., Mark, D. F., Takemura, K., and Danhara, T.: Identification and correlation of visible tephras in the Lake Suigetsu SG06 sedimentary archive, Japan: chronostratigraphic markers for synchronising of east Asian/west Pacific palaeoclimatic records across the last $150 \mathrm{ka}$, Quaternary Sci. Rev., 67, 121-137, https://doi.org/10.1016/j.quascirev.2013.01.026, 2013.

Snowball, I., Mellström, A., Ahlstrand, E., Haltia, E., Nilsson, A., Ning, W., Muscheler, R., and Brauer, A.: An estimate of postdepositional remanent magnetization lock-in depth in organic rich varved lake sediments, Global Planet. Change, 110, 264277, https://doi.org/10.1016/j.gloplacha.2013.10.005, 2013.

Stager, J. C., Mayewski, P. A., and Meeker, L. D.: Cooling cycles, Heinrich event 1, and the desiccation of Lake Victoria, Palaeogeogr. Palaeocl., 183, 169-178, https://doi.org/10.1016/s00310182(01)00468-0, 2002.

Stager, J. C., Ryves, D., Cumming, B. F., Meeker, L. D., and Beer, J.: Solar variability and the levels of Lake Victoria, East Africa, during the last millenium, J. Paleolimnol., 33, 243-251, https://doi.org/10.1007/s10933-004-4227-2, 2005.

Stebich, M., Brüchmann, C., Kulbe, T., and Negendank, J. F. W.: Vegetation history, human impact and climate change during 
the last 700 years recorded in annually laminated sediments of Lac Pavin, France, Rev. Palaeobot. Palyno., 133, 115-133, https://doi.org/10.1016/j.revpalbo.2004.09.004, 2005.

Stone, J. R. and Fritz, S. C.: Multidecadal drought and Holocene climate instability in the Rocky Mountains, Geology, 34, 409, https://doi.org/10.1130/g22225.1, 2006.

Striberger, J., Björck, S., Ingólfsson, Ó., Kjaer, K. H., Snowball, I., and Uvo, C. B.: Climate variability and glacial processes in eastern Iceland during the past 700 years based on varved lake sediments, Boreas, 40, 28-45, https://doi.org/10.1111/j.15023885.2010.00153.x, 2010.

Sullivan, D. G.: The discovery of Santorini Minoan tephra in western Turkey, Nature, 333, 552-554, https://doi.org/10.1038/333552a0, 1988.

Svensson, A., Andersen, K. K., Bigler, M., Clausen, H. B., Dahl-Jensen, D., Davies, S. M., Johnsen, S. J., Muscheler, R., Rasmussen, S. O., Röthlisberger, R., Steffensen, J. P., and Vinther, B. M.: The Greenland Ice Core Chronology 2005, 1542 ka. Part 2: Comparison to other records. Quaternary Sci. Rev., 25, 3246-3257, 2006.

Swierczynski, T., Lauterbach, S., Dulski, P., and Brauer, A.: Late Neolithic Mondsee Culture in Austria: living on lakes and living with flood risk?, Clim. Past, 9, 1601-1612, https://doi.org/10.5194/cp-9-1601-2013, 2013.

Thomas, E. K. and Briner, J. P.: Climate of the past millennium inferred from varved proglacial lake sediments on northeast Baffin Island, Arctic Canada, J. Paleolimnol., 41, 209-224, https://doi.org/10.1007/s10933-008-9258-7, 2008.

Thomas, E. K., McGrane, S., Briner, J. P., and Huang, Y.: Leaf wax $\delta 2 \mathrm{H}$ and varve-thickness climate proxies from proglacial lake sediments, Baffin Island, Arctic Canada, J. Paleolimnol., 48, 193-207, https://doi.org/10.1007/s10933-012-9584-7, 2012.

Tierney, J. E. and Russell, J. M.: Abrupt climate change in southeast tropical Africa influenced by Indian monsoon variability and ITCZ migration, Geophys. Res. Lett., 34, L15709, https://doi.org/10.1029/2007g1029508, 2007.

Tierney, J. E., Mayes, M. T., Meyer, N., Johnson, C., Swarzenski, P. W., Cohen, A. S., and Russell, J. M.: Late-twentiethcentury warming in Lake Tanganyika unprecedented since AD 500, Nat. Geosci., 3, 422-425, https://doi.org/10.1038/ngeo865, 2010.

Tierney, J. E., Russell, J. M., Huang, Y., Damste, J. S. S., Hopmans, E. C. and Cohen, A. S.: Northern Hemisphere Controls on Tropical Southeast African Climate During the Past 60,000 Years, Science, 322, 252-255, https://doi.org/10.1126/science.1160485, 2008.

Tlan, J., Brown, T. A., and Hul, F. S.: Comparison of varve and ${ }^{14} \mathrm{C}$ chronologies from Steel Lake, Minnesota, USA, Holocene, 15, 510-517, https://doi.org/10.1191/0959683605hl828rp, 2005.

Vasskog, K., Paasche, Ø., Nesje, A., Boyle, J. F., and Birks, H. J. B.: A new approach for reconstructing glacier variability based on lake sediments recording input from more than one glacier, Quaternary Res., 77, 192-204, https://doi.org/10.1016/j.yqres.2011.10.001, 2012.

Verschuren, D., Sinninghe Damsté, J. S., Moernaut, J., Kristen, I., Blaauw, M., Fagot, M., and Haug, G. H.: Half-precessional dynamics of monsoon rainfall near the East African Equator, Nature, 462, 637-641, https://doi.org/10.1038/nature08520, 2009.
Vinther, B. M., Clausen, H. B., Johnsen, S. J., Rasmussen, S. O., Andersen, K. K., Buchardt, S. L., Dahl-Jensen, D., Seierstad, I. K., Siggaard-Andersen, M.-L., Steffensen, J. P., Svensson, A. M., Olsen, J., and Heinemeier, J.: A synchronized dating of three Greenland ice cores throughout the Holocene. J. Geophys. Res., 111, D13102, https://doi.org/10.1029/2005JD006921, 2006.

Vogel, H., Wagner, B., Zanchetta, G., Sulpizio, R., and Rosén, P.: A paleoclimate record with tephrochronological age control for the last glacial-interglacial cycle from Lake Ohrid, Albania and Macedonia, J. Paleolimnol., 44, 295-310, https://doi.org/10.1007/s10933-009-9404-x, 2010a.

Vogel, H., Zanchetta, G., Sulpizio, R., Wagner, B. and Nowaczyk, N.: A tephrostratigraphic record for the last glacial-interglacial cycle from Lake Ohrid, Albania and Macedonia, J. Quat. Sci., 25, 320-338, https://doi.org/10.1002/jqs.1311, 2010 b.

Wagner, B., Lotter, A. F., Nowaczyk, N., Reed, J. M., Schwalb, A., Sulpizio, R., Valsecchi, V., Wessels, M., and Zanchetta, G.: A 40,000-year record of environmental change from ancient Lake Ohrid (Albania and Macedonia), J. Paleolimnol., 41, 407-430, https://doi.org/10.1007/s10933-008-9234-2, 2008.

Wagner, B., Aufgebauer, A., Vogel, H., Zanchetta, G., Sulpizio, R., and Damaschke, M.: Late Pleistocene and Holocene contourite drift in Lake Prespa (Albania/F.Y.R. of Macedonia/Greece), Quatern. Int., 274, 112-121, https://doi.org/10.1016/j.quaint.2012.02.016, 2012.

Wagner, B., Vogel, H., Zanchetta, G., and Sulpizio, R.: Environmental change within the Balkan region during the past ca. $50 \mathrm{ka}$ recorded in the sediments from lakes Prespa and Ohrid, Biogeosciences, 7, 3187-3198, https://doi.org/10.5194/bg-7-31872010, 2010.

Watts, W.: Vegetation history and palaeoclimate of the last glacial period of Lago Grande di Monticchio, southern Italy, Quaternary Sci. Rev., 15, 133-153, https://doi.org/10.1016/02773791(95)00093-3, 1996.

Werner, A.: NOAA/WDS Paleoclimatology - Lake Linne', Spitsbergen 20th Century Varved Sediment Data, NOAA National Centers for Environmental Information, available at: https:// www.ncdc.noaa.gov/paleo/study/8760 (last access: 15 september 2020), 2009.

Whitlock, C., Dean, W. E., Fritz, S. C., Stevens, L. R., Stone, J. R., Power, M. J., Rosenbaum, J. R., Pierce, K. L., and BrachtFlyr, B. B.: Holocene seasonal variability inferred from multiple proxy records from Crevice Lake, Yellowstone National Park, USA, Palaeogeogr. Palaeocl., 331-332, 90-103, https://doi.org/10.1016/j.palaeo.2012.03.001, 2012.

Williams, J. W., Grimm, E. C., Blois, J. L., Charles, D. F., Davis, E. B., Goring, S. J., Graham, R. W., Smith, A. J., Anderson, M., Arroyo-Cabrales, J., Ashworth, A. C., Betancourt, J. L., Bills, B. W., Booth, R. K., Buckland, P. I., Curry, B. B., Giesecke, T., Jackson, S. T., Latorre, C., Nichols, J., Purdum, T., Roth, R. E., Stryker, M. and Takahara, H.: The Neotoma Paleoecology Database, a multiproxy, international, community-curated data resource, Quaternary Res., 89, 156177, https://doi.org/10.1017/qua.2017.105, 2018.

Williamson, D., Thouveny, N., Hillaire-Marcel, C., Mondeguer, A., Taieb, M., Tiercelin, J.-J., and Vincens, A.: Chronological potential of palaeomagnetic oscillations recorded in late quaternary 
sediments from Lake Tanganyika, Quaternary Sci. Rev., 10, 351361, https://doi.org/10.1016/0277-3791(91)90036-t, 1991.

Wolff, C., Haug, G. H., Timmermann, A., Damste, J. S. S., Brauer, A., Sigman, D. M., Cane, M. A., and Verschuren, D.: Reduced Interannual Rainfall Variability in East Africa During the Last Ice Age, Science, 333, 743-747, https://doi.org/10.1126/science.1203724, 2011.

Wulf, S., Kraml, M., Brauer, A., Keller, J., and Negendank, J. F. W.: Tephrochronology of the $100 \mathrm{ka}$ lacustrine sediment record of Lago Grande di Monticchio (southern Italy), Quatern. Int., 122, 7-30, https://doi.org/10.1016/j.quaint.2004.01.028, 2004.

Wulf, S., Keller, J., Paterne, M., Mingram, J., Lauterbach, S., Opitz, S., Sottili, G., Giaccio, B., Albert, P. G., Satow, C., Tomlinson, E. L., Viccaro, M., and Brauer, A.: The 100-133 ka record of Italian explosive volcanism and revised tephrochronology of Lago Grande di Monticchio, Quaternary Sci. Rev., 58, 104-123, https://doi.org/10.1016/j.quascirev.2012.10.020, 2012.

Wulf, S., Ott, F., Słowiński, M., Noryśkiewicz, A. M., Dräger, N., Martin-Puertas, C., Czymzik, M., Neugebauer, I., Dulski, P., Bourne, A. J., Błaszkiewicz, M., and Brauer, A.: Tracing the Laacher See Tephra in the varved sediment record of the Trzechowskie palaeolake in central Northern Poland, Quaternary Sci. Rev., 76, 129-139, https://doi.org/10.1016/j.quascirev.2013.07.010, 2013.

Wulf, S., Dräger, N., Ott, F., Serb, J., Appelt, O., Guðmundsdóttir, E., van den Bogaard, C., Słowiński, M., Błaszkiewicz, M., and Brauer, A.: Holocene tephrostratigraphy of varved sediment records from Lakes Tiefer See (NE Germany) and Czechowskie (N Poland), Quaternary Sci. Rev., 132, 1-14, https://doi.org/10.1016/j.quascirev.2015.11.007, 2016.

Yamada, K., Kamite, M., Saito-Kato, M., Okuno, M., Shinozuka, Y., and Yasuda, Y.: Late Holocene monsoonalclimate change inferred from Lakes Ni-no-Megata and Sanno-Megata, northeastern Japan, Quatern. Int., 220, 122-132, https://doi.org/10.1016/j.quaint.2009.09.006, 2010.
Yu, Z. and Eicher, U.: Abrupt Climate Oscillations During the Last Deglaciation in Central North America, Science, 282, 22352238, https://doi.org/10.1126/science.282.5397.2235, 1998.

Żarczyński, M., Tylmann, W., and Goslar, T.: Multiple varve chronologies for the last 2000 years from the sediments of Lake Żabińskie (northeastern Poland) - Comparison of strategies for varve counting and uncertainty estimations, Quat. Geochronol., 47, 107-119, https://doi.org/10.1016/j.quageo.2018.06.001, 2018.

Zhou, A., Chen, F., Wang, Z., Yang, M., Qiang, M., and Zhang, J.: Temporal Change of Radiocarbon Reservoir Effect in Sugan Lake, Northwest China during the Late Holocene, Radiocarbon, 51, 529-535, https://doi.org/10.1017/s0033822200055909, 2009.

Zillén, L. M., Wastegård, S., and Snowball, I. F.: Calendar year ages of three mid-Holocene tephra layers identified in varved lake sediments in west central Sweden, Quaternary Sci. Rev., 21, 15831591, https://doi.org/10.1016/s0277-3791(02)00036-7, 2002.

Zolitschka, B.: Sedimentology, dating and palaeoclimatic interpretation of a $76.3 \mathrm{ka}$ record from Lago Grande di Monticchio, southern Italy, Quaternary Sci. Rev., 15, 101-112, https://doi.org/10.1016/0277-3791(95)00022-4, 1996.

Zolitschka, B., Brauer, A., Negendank, J. F. W., Stockhausen, H., and Lang, A.: Annually dated late Weichselian continental paleoclimate record from the Eifel, Germany, Geology, 28, 783, https://doi.org/10.1130/00917613(2000)28<783:adlwcp>2.0.co;2, 2000. 